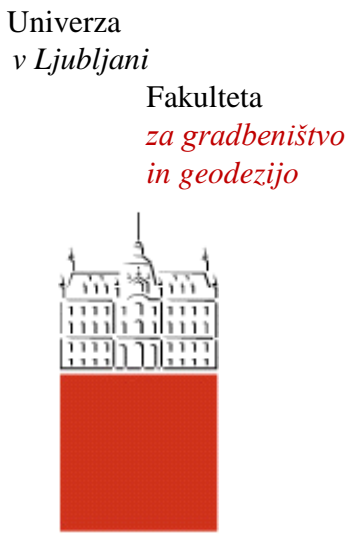

Jamova cesta 2

1000 Ljubljana, Slovenija

http://www3.fgg.uni-lj.si/

\section{DRUGG - Digitalni repozitorij UL FGG http://drugg.fgg.uni-lj.si/}

Ta članek je avtorjeva zadnja recenzirana različica, kot je bila sprejeta po opravljeni recenziji.

Prosimo, da se pri navajanju sklicujte na bibliografske podatke, kot je navedeno:
University
of Ljubljana

Faculty of Civil and Geodetic Engineering

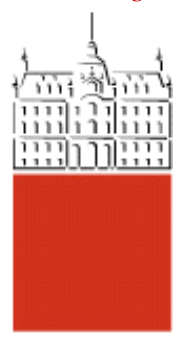

Jamova cesta 2 SI - 1000 Ljubljana, Slovenia http://www3.fgg.uni-lj.si/en/

DRUGG - The Digital Repository http://drugg.fgg.uni-lj.si/

This version of the article is author's manuscript as accepted for publishing after the review process.

When citing, please refer to the publisher's bibliographic information as follows:

Zupan, E., Saje, M., Zupan, D. 2009. The quaternion-based three-dimensional beam theory. Computer Methods in Applied Mechanics and Engineering 98,49-52: 3944-3956. DOI: 10.1016/j.cma.2009.09.002. 


\title{
The quaternion-based three-dimensional beam theory
}

\author{
E. Zupan, M. Saje and D. Zupan* \\ University of Ljubljana, Faculty of Civil and Geodetic Engineering, Jamova 2, \\ SI-1115 Ljubljana, Slovenia
}

\begin{abstract}
This paper presents the equations for the implementation of rotational quaternions in the geometrically exact three-dimensional beam theory. A new finite-element formulation is proposed in which the rotational quaternions are used for parametrization of rotations along the length of the beam. The formulation also satisfies the consistency condition that the equilibrium and the constitutive internal force and moment vectors are equal in its weak form. A strict use of the quaternion algebra in the derivation of governing equations and for the numerical solution is presented. Several numerical examples demonstrate the validity, performance and accuracy of the proposed approach.
\end{abstract}

Key words: quaternions, three-dimensional rotation, three-dimensional beam, interpolation of rotations

\section{Introduction}

In non-linear three-dimensional beam formulations, the deformed geometry of the beam is described by the deformed axis of the beam and by the rotation of the cross-sections. The configuration space of the beam thus consists of (i) the linear space of the position vector of the deformed axis, and (ii) the non-linear space of rotations of cross-sections, and is thus a non-linear manifold. The non-linearity of spatial rotations requires a special treatment, which makes the study of three-dimensional beams interesting and challenging.

Among suitable mathematical tools to treat the rotations are the Lie algebra, the Clifford algebra and the algebra of quaternions. The mathematical modelling of spatial rotations, their parametrization and linearization have been discussed in several publications, see, e.g. Argyris [1], Argyris and Poterasu

* Corresponding author. E-mail address: dejan.zupan@fgg.uni-lj.si 
[3], Atluri and Cazzani [4], and Géradin and Rixen [10]. While alternative non-rotation based three-dimensional beam formulations have aslo been proposed (see, e.g. [21]), finite rotations are taken to be the primary variables by most of authors. Battini and Pacoste [6], Cardona and Géradin [8], Crivelli and Felippa [9], Ibrahimbegović [12], Iura and Atluri [14], Jelenić and Saje [16], Nour-Omid and Rankin [20], Simo [28], Simo and Vu-Quoc [29], Zupan and Saje [34], to mention just a few, contributed to the development of the rotation-based finite-element beam formulations. The way the rotations are parametrized is in the beam theory crucial, because a particular selection of the parametrization has a strong impact on both the algorithm and the form of the tangent stiffness matrix. In the present paper we consider the so called 'geometrically exact finite-strain beam theory' (Reissner [24] and Simo [28]). In this approach the strain measures are fully complemental to the crosssectional stress resultants through the virtual work principle at the deformed state for any magnitude of displacements, rotations, and strains.

There is a number of possible ways of choosing the parameters describing spatial rotations. As the three independent parameters suffice to describe locally the spatial rotation, the three-parameter formulations have almost exclusively been used in beam theories. A particularly important representative of these parametrizations is the 'rotational vector' [1]. While the rotational vector possesses a clear geometrical meaning, which is an advantage, one of its disadvantages is that the discretization of rotations using a standard additive interpolation spoils the objectivity of rotational strain measures outside the interpolation points (Jelenić and Crisfield [15]). Jelenić and Crisfield [15] proposed a new formulation based on the linked interpolation of incremental local rotations which resolves the objectivity, but enhances the complexity of the formulation substantially. An alternative approach by Betsch and Steinmann [7] employs the nine-parameter description of rotations, in which the components of the director triad are interpolated. Their approach relaxes the orthogonality condition to hold only at the discretization points of the element. Several alternative interpolation strategies have also been discussed by Romero in [25] where the quaternions for the rotational update and the interpolation purposes are also presented.

A promising alternative for the parametrization of rotations is the four-parameter rotational quaternion. The use of rotational quaternions in beam theories has been so far limited to construct numerically stable and efficient local computational algorithms that avoid singularity problems and accelerate computations. An example of a widely used quaternion-based algorithm in beam formulations is the extraction of rotational parameters from the rotation matrix (Spurrier [30]). Yet the quaternions not only prove to be a numerically stable and computationally efficient tool for algebraic operations with rotations, but also offer an alternative description of rotations in four dimensions. The use of quaternions as the primary variables of the problem is now well 
spread in aerodynamics [22], robotics [33] and computer graphics [27]. Quaternions proved to be the most suitable parametrization of rotations in dynamics of rigid bodies; see the paper by Zupan and Saje [37] for further details. The theoretical background of the quaternion algebra and the related Clifford algebra is well established, see, e.g. the monographs by Ward [32] and Poreous [23]. McRobie and Lasenby [18] discuss advantages of employing the Clifford algebra, show crucial steps needed for the Simo and Vu-Quoc formulation [29]

and compare the steps of the Lie algebra to be replaced by equivalent simpler forms in terms of the Clifford algebra.

In the present paper, we fully abandon the rotation matrix concept and introduce the rotational quaternions as the only rotational unknowns of the problem. Thus not only the local computational algorithms, but also the equations of the beam are presented in terms of the quaternions. Strict use of quaternions results in formulations that differ considerably from the classical beam formulations, so the direct incorporation in existing computer programs is not possible without some modifications. However, the use of quaternions brings a number of advantages so that modifications are worth being done. It is shown that quaternion algebra provides a suitable and efficient tool both for the formulation of the continuum and discrete governing equations of the beam, and for the implementation of numerical algorithms. Furthermore, a novel, collocational-type of finite-element formulation is presented based on the consistent equilibrium at the chosen cross-sections requiring that the crosssectional stress resultants, obtained from the equilibrium equations and from the constitutive equations, are equilibrated.

\section{Quaternion parametrization of rotations}

Rotations and their application in computational mechanics have been extensively discussed in literature. Mathematically, rotations represent a multiplicative group of proper orthogonal operators. For the three-dimensional rotational operator to be expressed by the associated matrix, nine scalar components are needed. Not all of them are independent, in fact only three scalar values suffice to describe fully the rotation in the three-dimensional space. There exists a number of ways to parametrize the rotations, see, e.g. the comprehensive texts by Argyris [1], Atluri and Cazzani [4], and Géradin and Rixen [10]. It is well known that any choice of three parameters for describing spatial rotations results in a singularity. Our objective here is to employ a special fourparameter description of rotations known as the 'quaternion parametrization of rotations'. Because this parametrization uses a special algebra, called the algebra of quaternions, some mathematical fundamentals need to be presented first. 
The set of quaternions, $\mathbb{H}$, is formally defined as an abstract set of elements $\widehat{a}$, formed from scalars and vectors:

$$
\mathbb{H}=\left\{\widehat{a}=a_{0}+\vec{a}, \quad a_{0} \in \mathbb{R}, \vec{a} \in \mathbb{R}^{3}\right\}
$$

Three elementary operations are defined for any quaternions $\widehat{a}, \widehat{b} \in \mathbb{H}, \widehat{a}=$ $a_{0}+\vec{a}, \widehat{b}=b_{0}+\vec{b}$, and any scalar $\lambda \in \mathbb{R}$ :

- addition

$$
\begin{gathered}
(+): \mathbb{H} \times \mathbb{H} \rightarrow \mathbb{H} \\
\widehat{a}+\widehat{b}=\left(a_{0}+b_{0}\right)+(\vec{a}+\vec{b}),
\end{gathered}
$$

- scalar multiplication

$$
\begin{gathered}
(): \mathbb{R} \times \mathbb{H} \rightarrow \mathbb{H} \\
\lambda \widehat{a}=\lambda a_{0}+\lambda \vec{a},
\end{gathered}
$$

- and quaternion multiplication

$$
\begin{gathered}
(\circ): \mathbb{H} \times \mathbb{H} \rightarrow \mathbb{H} \\
\widehat{a} \circ \widehat{b}=\left(a_{0} b_{0}-\vec{a} \cdot \vec{b}\right)+\left(b_{0} \vec{a}+a_{0} \vec{b}+\vec{a} \times \vec{b}\right),
\end{gathered}
$$

where $(\cdot)$ and $(\times)$ denote the scalar and the cross-vector product, respectively, in vector space $\mathbb{R}^{3}$. The identity element for the quaternion multiplication is denoted by $\widehat{1}=1+\overrightarrow{0}$; thus $\widehat{a} \circ \widehat{1}=\widehat{1} \circ \widehat{a}=\widehat{a}$ for any $\widehat{a} \in \mathbb{H}$.

The set of the quaternions with the addition and the scalar multiplication is the four-dimensional linear space over $\mathbb{R}$ and is therefore isomorphic to $\mathbb{R}^{4}$. The quaternion multiplication (3) is associative, but not commutative, which makes the set of quaternions $\mathbb{H}$ an associative non-commutative algebra.

The quaternions with a zero scalar part are called pure quaternions. They constitute a three-dimensional linear subspace of $\mathbb{H}$ which is isomorphic to $\mathbb{R}^{3}$. Therefore, we can identify a pure quaternion with its vector part

$$
0+\vec{a} \equiv \vec{a}
$$

We further introduce

- conjugated quaternion

$$
\widehat{a}^{*}=a_{0}-\vec{a}
$$

- quaternion norm

$$
|\widehat{a}|=\sqrt{\widehat{a} \circ \widehat{a}^{*}}=\sqrt{a_{0}^{2}+|\vec{a}|^{2}}
$$


where $|\vec{a}|=\sqrt{\vec{a} \cdot \vec{a}}$ is Euclidean vector norm in the three-dimensional vector space,

- angle between two quaternions $\widehat{a}, \widehat{b} \in \mathbb{H}$

$$
\cos \lambda=\frac{a_{0} b_{0}+\vec{a} \cdot \vec{b}}{|\widehat{a}||\hat{b}|},
$$

- and inverse of a quaternion with the non-zero norm

$$
\widehat{a}^{-1}=\frac{\widehat{a}^{*}}{|\widehat{a}|} .
$$

When applied to a pure quaternion, the definition of the conjugated quaternion gives

$$
\widehat{a}^{*}=-\widehat{a} \quad \Longleftrightarrow \quad \widehat{a}=0+\vec{a} .
$$

From the above listed definitions, it can easily be shown that every quaternion can also be written in the polar form:

$$
\widehat{a}=|\widehat{a}|(\cos \vartheta+\vec{n} \sin \vartheta)
$$

where $\vec{n}$ is a unit vector. When $|\widehat{a}|=1$, one easily identifies $\vec{n}$ as the axis of rotation with $\vartheta$ being the rotational angle. As discussed above, the quaternion multiplication is not commutative. Hence, for a given unit quaternion $\widehat{q}(|\widehat{q}|=$ 1 ) and an arbitrary quaternion $\widehat{x}$, we can introduce two linear operators

- left multiplication

$$
\phi_{L}(\widehat{q}): \widehat{x} \longmapsto \widehat{q} \circ \widehat{x}
$$

- and right multiplication

$$
\phi_{R}(\widehat{q}): \widehat{x} \longmapsto \widehat{x} \circ \widehat{q}
$$

For both $\phi_{L}$ and $\phi_{R}$ we can prove that they conserve length, angle, and orientation of $\widehat{x}$ [32]. Therefore $\phi_{L}$ and $\phi_{R}$ represent rotations in the four-dimensional space of quaternions. However, neither of them represents rotations in three dimensions, because they do not map pure quaternions (vectors) into pure quaternions (vectors). Yet it is easy to find a composite of the two operators that also fulfils the condition of mapping pure quaternion into a pure quaternion. Recalling that the composite of two rotations in four dimensions is again a four-dimensional rotation, we define a new rotational operator, $\mathcal{Q}$,

$$
\begin{aligned}
& \mathcal{Q}(\widehat{q})=\phi_{R}\left(\widehat{q}^{*}\right) \phi_{L}(\widehat{q})=\phi_{L}(\widehat{q}) \phi_{R}\left(\widehat{q}^{*}\right) \\
& \mathcal{Q}(\widehat{q}): \widehat{x} \longmapsto \widehat{q} \circ \widehat{x} \circ \widehat{q}^{*},
\end{aligned}
$$

which conserves pure quaternions. The proof that $\mathcal{Q}(0+\vec{x})$ results in a pure quaternion is straightforward and therefore omitted here. Because the axes of 
the two rotations are colinear, the angle of rotation of the composed rotation, $\mathcal{Q}=\phi_{R}\left(\widehat{q}^{*}\right) \phi_{L}(\widehat{q})$, is twice the angle of a single rotation $\phi_{R}\left(\widehat{q}^{*}\right)$ or $\phi_{L}(\widehat{q})$. Thus a unit quaternion $\widehat{q}$ can be expressed in its polar form as

$$
\widehat{q}=\cos \frac{\vartheta}{2}+\vec{n} \sin \frac{\vartheta}{2}
$$

Due to its direct relation to the axis and the angle of rotation, expression (9) is often taken as the definition of the rotational quaternion.

In the numerical implementation, the matrix form of quaternions is often more appropriate, yet it requires the introduction of the basis into the space of quaternions $\mathbb{H}$. The generalization of Euclidean orthonormal base vectors in $\mathbb{R}^{3},\left\{\vec{g}_{1}, \vec{g}_{2}, \vec{g}_{3}\right\}$, into $\mathbb{H}$ results in $\widehat{g}_{i}=0+\vec{g}_{i}, i=1,2,3$. These vectors uniquely represent only pure quaternions, hence the basis $\left\{\widehat{g}_{1}, \widehat{g}_{2}, \widehat{g}_{3}\right\}$ spans the three-dimensional subspace of pure quaternions. The fourth base quaternion is then naturally taken to be $\widehat{g}_{0}=1+\overrightarrow{0}=\widehat{1}$. It is easy to show that $\left\{\widehat{g}_{0}, \widehat{g}_{1}, \widehat{g}_{2}, \widehat{g}_{3}\right\}$ are orthonormal. With respect to $\left\{\widehat{g}_{0}, \widehat{g}_{1}, \widehat{g}_{2}, \widehat{g}_{3}\right\}$, any quaternion in $\mathbb{H}$ can be uniquely expressed as a linear combination

$$
\widehat{a}=a_{0} \widehat{g}_{0}+a_{1} \widehat{g}_{1}+a_{2} \widehat{g}_{2}+a_{3} \widehat{g}_{3}
$$

The set of quaternions $\left\{\widehat{g}_{0}, \widehat{g}_{1}, \widehat{g}_{2}, \widehat{g}_{3}\right\}$ will be called the quaternion basis.

It is found convenient to introduce the following matrix notation. The scalar components $a_{i}, i=0,1,2,3$, of a quaternion $\widehat{a}$, are described by the one-column matrix

$$
\widehat{\boldsymbol{a}}=\left[\begin{array}{llll}
a_{0} & a_{1} & a_{2} & a_{3}
\end{array}\right]^{T} .
$$

Similarly, the linear operators on the space of quaternions can be represented by $4 \times 4$ matrices. Considering the basis $\left\{\widehat{g}_{0}, \widehat{g}_{1}, \widehat{g}_{2}, \widehat{g}_{3}\right\}$ yields the linear maps (6)-(7) in their matrix representation as:

$$
\boldsymbol{\Phi}_{L}(\widehat{\boldsymbol{q}})=\left[\begin{array}{cccc}
q_{0} & -q_{1} & -q_{2} & -q_{3} \\
q_{1} & q_{0} & -q_{3} & q_{2} \\
q_{2} & q_{3} & q_{0} & -q_{1} \\
q_{3} & -q_{2} & q_{1} & q_{0}
\end{array}\right], \quad \boldsymbol{\Phi}_{R}(\widehat{\boldsymbol{q}})=\left[\begin{array}{cccc}
q_{0}-q_{1} & -q_{2} & -q_{3} \\
q_{1} & q_{0} & q_{3} & -q_{2} \\
q_{2}-q_{3} & q_{0} & q_{1} \\
q_{3} & q_{2} & -q_{1} & q_{0}
\end{array}\right],
$$

where $\widehat{\boldsymbol{q}}=\left[\begin{array}{llll}q_{0} & q_{1} & q_{2} & q_{3}\end{array}\right]^{T}$. It is easy to check that both $\boldsymbol{\Phi}_{L}$ and $\boldsymbol{\Phi}_{R}$ are proper orthogonal matrices, which shows that they are elements of a special orthogonal group $S O(4)$, and confirms that they can be identified with rotations in 
$\mathbb{R}^{4}$. Introducing the above given matrix notations into equations (6)-(7) gives

$$
\begin{aligned}
& \widehat{\boldsymbol{q}} \circ \widehat{\boldsymbol{x}}=\boldsymbol{\Phi}_{L}(\widehat{\boldsymbol{q}}) \widehat{\boldsymbol{x}} \\
& \widehat{\boldsymbol{x}} \circ \widehat{\boldsymbol{q}}=\boldsymbol{\Phi}_{R}(\widehat{\boldsymbol{q}}) \widehat{\boldsymbol{x}} .
\end{aligned}
$$

Note that equations (11)-(12) also hold true for any non-unit quaternion $\widehat{\boldsymbol{q}}$, but the matrices $\boldsymbol{\Phi}_{L}(\widehat{\boldsymbol{q}})$ and $\boldsymbol{\Phi}_{R}(\widehat{\boldsymbol{q}})$ are then not orthogonal. Consequently, equations (11)-(12) provide us with the general rule for expressing the quaternion product by the matrix product. There is another issue of interest that directly follows from (10): the multiplication of the matrices $\boldsymbol{\Phi}_{R}\left(\widehat{\boldsymbol{q}}^{*}\right)$ and $\boldsymbol{\Phi}_{L}(\widehat{\boldsymbol{q}})$ gives a $4 \times 4$ matrix

$$
\mathbf{Q}=\boldsymbol{\Phi}_{R}\left(\widehat{\boldsymbol{q}}^{*}\right) \boldsymbol{\Phi}_{L}(\widehat{\boldsymbol{q}})=\left[\begin{array}{ll}
1 & \mathbf{0}_{1 \times 3} \\
\mathbf{0}_{3 \times 1} & \mathbf{R}
\end{array}\right]
$$

with the submatrix $\mathbf{R}$ being the standard rotation matrix in the three-dimensional vector space (see, e.g. [29, Equation (B.4)] ):

$$
\mathbf{R}=2\left[\begin{array}{lll}
q_{0}^{2}+q_{1}^{2}-\frac{1}{2} & q_{1} q_{2}-q_{0} q_{3} & q_{1} q_{3}+q_{0} q_{2} \\
q_{1} q_{2}+q_{0} q_{3} & q_{0}^{2}+q_{2}^{2}-\frac{1}{2} & q_{2} q_{3}-q_{0} q_{1} \\
q_{1} q_{3}-q_{0} q_{2} & q_{2} q_{3}+q_{0} q_{1} & q_{0}^{2}+q_{3}^{2}-\frac{1}{2}
\end{array}\right]
$$

From its matrix representation (13) it is now evident that operator $\mathcal{Q}$ maps a pure quaternion into a pure quaternion with the vector part being rotated only:

$$
\mathbf{Q}\left[\begin{array}{l}
0 \\
\boldsymbol{a}
\end{array}\right]=\left[\begin{array}{ll}
1 & \mathbf{0}^{T} \\
\mathbf{0} & \boldsymbol{R}
\end{array}\right]\left[\begin{array}{l}
0 \\
\boldsymbol{x}
\end{array}\right]=\left[\begin{array}{c}
0 \\
\boldsymbol{R} \boldsymbol{x}
\end{array}\right] .
$$

\section{Geometry of the three-dimensional beam}

\subsection{Basic definitions}

An arbitrary configuration of the three-dimensional beam is uniquely described by the position vector $\vec{r}(x)$ of the line of centroids and by the family of orthonormal base vectors $\left\{\vec{G}_{1}(x), \vec{G}_{2}(x), \vec{G}_{3}(x)\right\}$, with $\vec{G}_{2}$ and $\vec{G}_{3}$ spanning the planes of cross-sections and $\vec{G}_{1}$ being the normal vector of the cross-section. In general all the quantities are dependent on parameter $x \in[0, L]$, the arclength of the line of centroids in the initial configuration. We assume that the cross-sections are rigid, thus conserving their shape during deformation. In order to describe the deformation of the beam with respect to the physical space, 
we introduce an orthonormal triad $\left\{\vec{g}_{1}, \vec{g}_{2}, \vec{g}_{3}\right\}$ of fixed vectors together with the reference point $\mathcal{O}$, which define the global coordinate system $(X, Y, Z)$.

An arbitrary vector, $\vec{a}$, can be expressed with respect to either of the two vector bases

$$
\vec{a}=a_{g 1} \vec{g}_{1}+a_{g 2} \vec{g}_{2}+a_{g 3} \vec{g}_{3}=a_{G 1} \vec{G}_{1}+a_{G 2} \vec{G}_{2}+a_{G 3} \vec{G}_{3} .
$$

The scalar components in (14) may also be represented by one-column matrices with three components

$$
\boldsymbol{a}_{g}=\left[\begin{array}{lll}
a_{g 1} & a_{g 2} & a_{g 3}
\end{array}\right]^{T}, \quad \boldsymbol{a}_{G}=\left[\begin{array}{lll}
a_{G 1} & a_{G 2} & a_{G 3}
\end{array}\right]^{T},
$$

where index $g$ or $G$ denotes the corresponding basis. The relationship between the two representations is given by the rotational operator $\mathcal{R}(x)$, which maps $\left\{\vec{g}_{1}, \vec{g}_{2}, \vec{g}_{3}\right\}$ into $\left\{\vec{G}_{1}, \vec{G}_{2}, \vec{G}_{3}\right\}$ :

$$
\vec{G}_{i}=\mathcal{R} \vec{g}_{i}, \quad i=1,2,3 .
$$

Operator $\mathcal{R}(x)$ is a linear operator on the three-dimensional Euclidean space and represents the rotation between the two bases. Its matrix representation is obtained by expressing vectors $\vec{G}_{i}$ as the linear combination of base vectors $\left\{\vec{g}_{1}, \vec{g}_{2}, \vec{g}_{3}\right\}:$

$$
\begin{aligned}
\vec{G}_{i} & =\mathrm{R}_{1 i} \vec{g}_{1}+\mathrm{R}_{2 i} \vec{g}_{2}+\mathrm{R}_{3 i} \vec{g}_{3}, \quad i=1,2,3 \\
\mathbf{R} & =\left[\mathrm{R}_{j i}\right], \quad i, j=1,2,3 .
\end{aligned}
$$

Inserting (16) into (14) gives the matrix relationship between the two onecolumn representations:

$$
\boldsymbol{a}_{g}=\mathbf{R} \boldsymbol{a}_{G} .
$$

In order to apply the quaternion algebra for the description of rotations, we

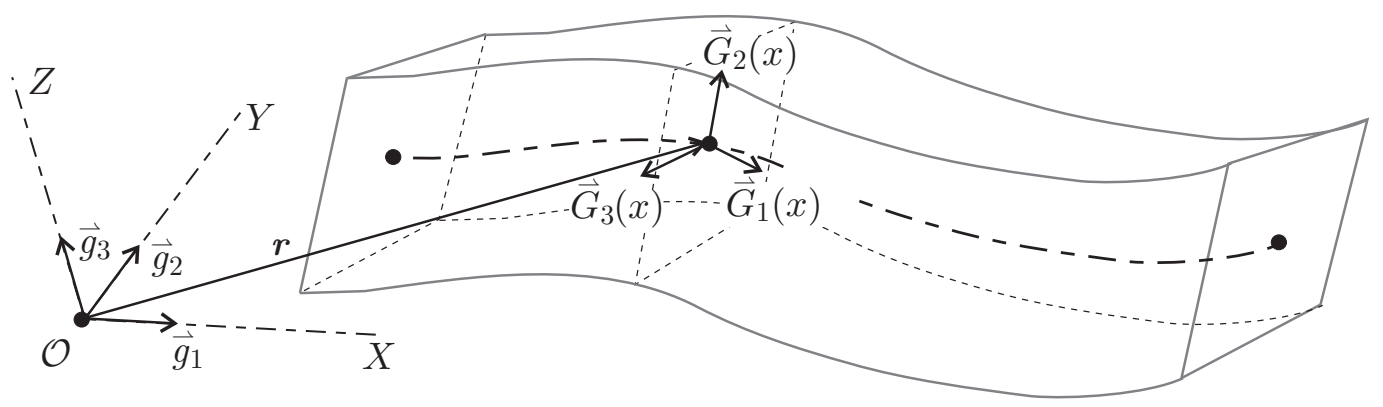

Fig. 1. Model of the three-dimensional beam. 
need to extend the space of rotations into four dimensions. As already explained, a direct generalization is achieved by (i) adding quaternion $\widehat{1}$ to the three-dimensional basis, and (ii) substituting the three-dimensional vector basis with the pure quaternions. Thus, the global and the local bases of the beam in the quaternion space become

$$
\left\{\widehat{g}_{0}, \widehat{g}_{1}, \widehat{g}_{2}, \widehat{g}_{3}\right\} \quad \text { and } \quad\left\{\widehat{G}_{0}, \widehat{G}_{1}, \widehat{G}_{2}, \widehat{G}_{3}\right\}
$$

here $\widehat{g}_{0}=\widehat{G}_{0}=1+\overrightarrow{0}=\widehat{1}$ and $\widehat{g}_{i}=0+\vec{g}_{i}, \widehat{G}_{i}=0+\vec{G}_{i}, i=1,2,3$. In contrast to $\vec{G}_{i}, i=1,2,3, \widehat{G}_{0}$ is not dependent on parameter $x$. One-column quaternion representations of vectors then follow as:

$$
\widehat{\boldsymbol{a}}_{g}=\left[\begin{array}{llll}
0 & a_{g 1} & a_{g 2} & a_{g 3}
\end{array}\right]^{T}, \quad \widehat{\boldsymbol{a}}_{G}=\left[\begin{array}{llll}
0 & a_{G 1} & a_{G 2} & a_{G 3}
\end{array}\right]^{T} .
$$

The rotational operator $\mathcal{R}$ is in the quaternion space identified with linear map $\mathcal{Q}$. Consequently, the quaternion version of equation (15) reads

$$
\widehat{G}_{i}=\mathcal{Q} \widehat{g}_{i}=\widehat{q} \circ \widehat{g}_{i} \circ \widehat{q}^{*}, \quad i=1,2,3 .
$$

The coordinate transformation (17) can now be written in the quaternion notation as

$$
\widehat{\boldsymbol{a}}_{g}=\mathbf{Q} \widehat{\boldsymbol{a}}_{G}=\widehat{\boldsymbol{q}} \circ \widehat{\boldsymbol{a}}_{G} \circ \widehat{\boldsymbol{q}}^{*} .
$$

The inverse of the above relation must consider the fact that operator $\mathcal{Q}$ and its matrix $\mathbf{Q}$ are orthogonal. This yields

$$
\begin{aligned}
\widehat{\boldsymbol{a}}_{G} & =\mathbf{Q}^{T} \widehat{\boldsymbol{a}}_{g}=\boldsymbol{\phi}_{L}^{T}(\widehat{\boldsymbol{q}}) \boldsymbol{\phi}_{R}^{T}\left(\widehat{\boldsymbol{q}}^{*}\right) \widehat{\boldsymbol{a}}_{g}=\boldsymbol{\phi}_{R}(\widehat{\boldsymbol{q}}) \boldsymbol{\phi}_{L}\left(\widehat{\boldsymbol{q}}^{*}\right) \widehat{\boldsymbol{a}}_{g} \\
& =\widehat{\boldsymbol{q}}^{*} \circ \widehat{\boldsymbol{a}}_{g} \circ \widehat{\boldsymbol{q}}
\end{aligned}
$$

Note that the rotational quaternion has identical representations in both bases, i.e. $\widehat{\boldsymbol{q}}_{G}=\widehat{\boldsymbol{q}}_{g}=\widehat{\boldsymbol{q}}$.

\subsection{Derivative and variation of rotations}

The derivative of base vectors $\vec{G}_{i}$ with respect to parameter $x$ is essential in any beam theory. If equation (15) is differentiated and the result expressed with respect to the same basis, we have

$$
\vec{G}_{i}^{\prime}=\mathcal{R}^{\prime} \vec{g}_{i}=\mathcal{R}^{\prime} \mathcal{R}^{T} \vec{G}_{i}
$$

where $\Omega=\mathcal{R}^{\prime} \mathcal{R}^{T}$ is a physically sound measure of curvature. When the parametrization with the rotational vector is used, the form of equation (21) becomes non-trivial. In contrast, when rotational quaternions are used for the parametrization, the compact form of (21) is conserved (see, e.g. [10], [22]). 
The quaternion analogy to (21) can easily be derived. Starting from equation (18) and taking the first derivative with respect to $x$ yields

$$
\begin{aligned}
\widehat{G}_{i}^{\prime} & =\widehat{q}^{\prime} \circ \widehat{g}_{i} \circ \widehat{q}^{*}+\widehat{q} \circ \widehat{g}_{i} \circ \widehat{q}^{* \prime} \\
& =\widehat{q}^{\prime} \circ \widehat{q}^{*} \circ \widehat{G}_{i}+\widehat{G}_{i} \circ \widehat{q} \circ \widehat{q}^{* \prime} .
\end{aligned}
$$

For the rotational quaternions we have

$$
\widehat{q} \circ \widehat{q}^{*}=\widehat{q}^{*} \circ \widehat{q}=\widehat{1}=1+\overrightarrow{0} .
$$

The differentiation of the above equation with respect to $x$ gives

$$
\begin{aligned}
\widehat{q}^{\prime} \circ \widehat{q}^{*}+\widehat{q} \circ \widehat{q}^{* \prime} & =\widehat{0}, \\
\widehat{q} \circ \widehat{q}^{* \prime} & =-\widehat{q}^{\prime} \circ \widehat{q}^{*}=-\left(\widehat{q} \circ \widehat{q}^{* \prime}\right)^{*} .
\end{aligned}
$$

Due to (4) $\widehat{q} \circ \widehat{q}^{* \prime}$ is a pure quaternion. $\widehat{G}_{i}, i=1,2,3$, is a pure quaternion by the definition. When the two facts are considered, equation (22) simplifies into

$$
\widehat{G}_{i}^{\prime}=2 \widehat{q}^{\prime} \circ \widehat{q}^{*} \circ \widehat{G}_{i} .
$$

We are now able to identify a pure quaternion $\widehat{\kappa}$ as

$$
\widehat{\kappa}=2 \widehat{q}^{\prime} \circ \widehat{q}^{*}
$$

which is the quaternion representation of the curvature vector. In classical mechanics the vector part of this quantity is interpreted as the axial vector of the skew-symmetric operator $\mathcal{R}^{\prime} \mathcal{R}^{T}$. See [1] and [4] for further details.

Because $\widehat{\kappa}=0+\vec{\kappa}$ is a pure quaternion, equation (24) can also be written as

$$
\widehat{G}_{i}^{\prime}=0+\vec{\kappa} \times \vec{G}_{i}
$$

As the scalar part in (26) vanishes, it suffices to observe its vector part only. It is convenient to introduce the notation

$$
[\widehat{a}]_{\mathbb{R}^{3}}=\left[a_{0}+\vec{a}\right]_{\mathbb{R}^{3}}=\vec{a}
$$

for the projection of a quaternion onto the three-dimensional Euclidean space. The projection of equation (26) onto the three-dimensional space then reads

$$
\vec{G}_{i}^{\prime}=2\left[\widehat{q}^{\prime} \circ \widehat{q}^{*}\right]_{\mathbb{R}^{3}} \times \vec{G}_{i} .
$$

In what follows we need the component form of equation (25). Following the rules in equations (18) and (19), the representations of $\widehat{\kappa}$ with respect to the two bases read

$$
\widehat{\boldsymbol{\kappa}}_{g}=2 \widehat{\boldsymbol{q}}^{\prime} \circ \widehat{\boldsymbol{q}}^{*} \quad \widehat{\boldsymbol{\kappa}}_{G}=2 \widehat{\boldsymbol{q}}^{*} \circ \widehat{\boldsymbol{q}}^{\prime} .
$$


It is well known that the variation of rotations needs to be performed carefully as the three-dimensional rotations are not additive. One of the important technical advantages of the quaternion parametrization of rotations is in a considerably simpler linearization. As the rotational quaternion is the basic unknown of the problem, its variation is an arbitrary quaternion, here denoted by $\delta \widehat{\boldsymbol{q}}$. However, such a linear change of the rotational quaternion has not a unit norm, which implies that it is not directly applicable as the increment of a rotational quaternion. An appropriate solution of this problem can be obtained by observing the variation of base vector $\widehat{G}_{i}$ which gives

$$
\delta \widehat{G}_{i}=2 \delta \widehat{q} \circ \widehat{q}^{*} \circ \widehat{G}_{i}
$$

where $2 \delta \widehat{q} \circ \widehat{q}^{*}$ is a pure, yet a non-unit quaternion. We will use the notation

$$
\delta \widehat{\vartheta}=2 \delta \widehat{q} \circ \widehat{q}^{*}
$$

introducing the measure for the rate of change of the basis $\widehat{G}_{i}$ due to the variation of the quaternion. The advantage of $\delta \widehat{\vartheta}$ compared to $\delta \widehat{q}$ is that its projection onto the set of rotational quaternions can be easily found. Since

$\delta \widehat{\vartheta}=0+\delta \vec{\vartheta}$, the corresponding unit rotational quaternion $\widehat{q}$ can be defined from the polar form (9) as

$$
\widehat{q}(\delta \hat{\vartheta})=\cos \frac{\delta \vartheta}{2}+\frac{\delta \vec{\vartheta}}{\delta \vartheta} \sin \frac{\delta \vartheta}{2}
$$

where $\delta \vartheta$ denotes the norm of $\delta \widehat{\vartheta}$. Please observe the analogy with the standard variation of the rotational vector where $\delta \vec{G}_{i}=\delta \vec{\vartheta} \times \vec{G}_{i}$. The implementation of (31) that avoids singularities for small values of $|\delta \vartheta|$ (less than $\pi / 4$ ) in numerical computations is presented in Appendix A.

\section{Strain measures and stress resultants}

The geometrically exact finite-strain beam theory introduces two strain vectors [24]: (i) the translational strain vector $\vec{\gamma}$, and (ii) the rotational strain vector $\vec{\kappa}$. When expressed with respect to the base vectors $\vec{G}_{i}$, their components have sound physical interpretation: $\gamma_{G 1}$ is the extensional strain, and $\gamma_{G 2}, \gamma_{G 3}$ are shear strains; similarly, $\kappa_{G 1}$ is the torsional strain, and $\kappa_{G 2}, \kappa_{G 3}$ are the bending strains (curvatures).

The relations between the strain, displacement and rotational vectors are derived from the condition that the strains and stresses are consistent with the virtual work principle for any internal forces and any magnitude of deformation. This condition yields the following relationships between the variations of 
kinematic vector variables $(\vec{r}, \vec{\vartheta})$ and the relative variations of strain vectors $(\vec{\gamma}, \vec{\kappa}):$

$$
\begin{aligned}
& \delta(\vec{\gamma})_{\text {rel }}=\delta \vec{r}^{\prime}-\delta \vec{\vartheta} \times \vec{r}^{\prime} \\
& \delta(\vec{\kappa})_{\text {rel }}=\delta \vec{\vartheta}^{\prime}
\end{aligned}
$$

where $(\cdot)_{\text {rel }}$ is the relative variation of the vector with respect to basis $\vec{G}_{i}$, defined as

$$
\delta(\vec{u})_{\mathrm{rel}}=\delta u_{G 1} \vec{G}_{1}+\delta u_{G 2} \vec{G}_{2}+\delta u_{G 3} \vec{G}_{3}=\delta \vec{u}-\delta \vec{\vartheta} \times \vec{u} .
$$

See the paper of Zupan and Saje [34] for further details. According to (34), $\delta \vec{r}^{\prime}-\delta \vec{\vartheta} \times \vec{r}^{\prime}$ is the relative variation of vector $\vec{r}^{\prime}$; therefore, the integration of equation (32) gives

$$
\vec{\gamma}=\vec{r}^{\prime}+\vec{c}
$$

where $\vec{c}$ is a variational constant, given in the initial configuration of the beam. Although equation (33) looks simple, its integration in terms of rotational matrices and vectors is a demanding task (the details of the integration were presented by Reissner [24] and Ibrahimbegovic [13]). The integration is much easier using the quaternion algebra. This will become clear through the following derivation. The derivative of equation (30) with respect to $x$ gives

$$
\delta \widehat{\vartheta}^{\prime}=2 \delta \widehat{q}^{\prime} \circ \widehat{q}^{*}+2 \delta \widehat{q} \circ \widehat{q}^{* \prime},
$$

while the variation of equation (25) yields

$$
\begin{aligned}
\delta \widehat{\kappa} & =2 \delta \widehat{q}^{\prime} \circ \widehat{q}^{*}+2 \widehat{q}^{\prime} \circ \delta \widehat{q}^{*} \\
2 \delta \widehat{q}^{\prime} \circ \widehat{q}^{*} & =\delta \widehat{\kappa}-2 \widehat{q}^{\prime} \circ \delta \widehat{q}^{*} .
\end{aligned}
$$

Inserting (37) into (36) results in

$$
\begin{aligned}
\delta \widehat{\vartheta}^{\prime} & =\delta \widehat{\kappa}-2 \widehat{q}^{\prime} \circ \delta \widehat{q}^{*}+2 \delta \widehat{q} \circ \widehat{q}^{* \prime} \\
& =\delta \widehat{\kappa}-2 \widehat{q}^{\prime} \circ \widehat{q}^{*} \circ \widehat{q} \circ \delta \widehat{q}^{*}+2 \delta \widehat{q} \circ \widehat{q}^{*} \circ \widehat{q} \circ \widehat{q}^{* \prime} \\
& =\delta \widehat{\kappa}-\frac{1}{2} \widehat{\kappa} \circ \delta \widehat{\vartheta}^{*}+\frac{1}{2} \delta \widehat{\vartheta} \circ \widehat{\kappa}^{*} .
\end{aligned}
$$

Since both $\widehat{\kappa}$ and $\delta \widehat{\vartheta}$ are pure quaternions, we have

$$
\delta \widehat{\vartheta}^{\prime}=0+\delta \vec{\kappa}-\delta \vec{\vartheta} \times \vec{\kappa}=0+\delta(\vec{\kappa})_{\text {rel }} .
$$

The projection of equation (38) onto $\mathbb{R}^{3}$ equals (33), which proves the fact that the integrated form of equation (33) in terms of quaternions reads

$$
\widehat{\kappa}=2 \widehat{q}^{\prime} \circ \widehat{q}^{*}+\widehat{d}
$$


where $\widehat{d}$ is a variational constant given in the initial configuration of the beam.

Equations (35) and (39) were derived in an abstract form. For the computational purposes the component form is more convenient. To this aim it is suitable to choose a fixed basis $(g)$ to represent $\vec{r}, \widehat{r}$ and $\widehat{q}$, and a local basis $(G)$ for $\vec{\gamma}$ and $\widehat{\kappa}$. Equations (35) and (39) then take the matrix form

$$
\begin{aligned}
\boldsymbol{\gamma}_{G} & =\left[\widehat{\boldsymbol{q}}^{*}(x) \circ \widehat{\boldsymbol{r}}_{g}^{\prime}(x) \circ \widehat{\boldsymbol{q}}(x)\right]_{\mathbb{R}^{3}}+\boldsymbol{c}_{G} \\
\widehat{\boldsymbol{\kappa}}_{G} & =2 \widehat{\boldsymbol{q}}^{*} \circ \widehat{\boldsymbol{q}}^{\prime}+\widehat{\boldsymbol{d}}_{G}
\end{aligned}
$$

In the above equations, the index at $\widehat{\boldsymbol{q}}$ denoting the related basis is omitted since the componential representation is identical in the two bases.

It is found convenient to present the equilibrium equations of the beam with respect to the fixed basis:

$$
\begin{aligned}
\boldsymbol{n}_{g}(x) & =-\boldsymbol{N}_{g}^{\prime}(x) \\
\boldsymbol{m}_{g}(x) & =-\boldsymbol{M}_{g}^{\prime}(x)-\boldsymbol{r}_{g}^{\prime}(x) \times \boldsymbol{N}_{g}(x)
\end{aligned}
$$

Here, $\boldsymbol{N}_{g}$ and $\boldsymbol{M}_{g}$ are the stress resultants of the cross-section, i.e. the resultant force and the resultant moment vector. $\boldsymbol{n}_{g}$ and $\boldsymbol{m}_{g}$ are the external distributed force and moment vectors per unit of the undeformed length of the axis. It is obvious from equations (42)-(43) that the stress resultants depend on the external distributed force and moment vectors and on the deformed shape of the axis. On the other hand, the stress resultant vectors with respect to the local bases are related to strains by the constitutive equations given as

$$
\begin{aligned}
\boldsymbol{N}_{G} & =\mathcal{C}_{N}\left(\boldsymbol{\gamma}_{G}, \boldsymbol{\kappa}_{G}\right) \\
\boldsymbol{M}_{G} & =\mathcal{C}_{M}\left(\boldsymbol{\gamma}_{G}, \boldsymbol{\kappa}_{G}\right) .
\end{aligned}
$$

Operators $\mathcal{C}_{N}$ and $\mathcal{C}_{M}$ must be invariant under superimposed rigid-body motions and at least once differentiable with respect to $\boldsymbol{\gamma}_{G}$ and $\boldsymbol{\kappa}_{G}$, but are otherwise arbitrary. The condition that the constitutive-based stress resultants (44)-(45) should be equal to the equilibrium-based stress resultants obtained from (42)-(43) is imposed in the form

$$
\begin{aligned}
{\left[\widehat{\boldsymbol{q}}^{*} \circ \widehat{\boldsymbol{N}}_{g} \circ \widehat{\boldsymbol{q}}\right]_{\mathbb{R}^{3}} } & =\mathcal{C}_{N}\left(\boldsymbol{\gamma}_{G}, \boldsymbol{\kappa}_{G}\right) \\
\widehat{\boldsymbol{q}}^{*} \circ \widehat{\boldsymbol{M}}_{g} \circ \widehat{\boldsymbol{q}} & =\widehat{\mathcal{C}}_{M}\left(\boldsymbol{\gamma}_{G}, \boldsymbol{\kappa}_{G}\right),
\end{aligned}
$$

where the hat $\widehat{\bullet}$ over $\widehat{\boldsymbol{N}}_{g}$ and $\widehat{\boldsymbol{M}}_{g}$ denotes the expansion of these vectors to pure quaternions. Similarly, $\widehat{\mathcal{C}}_{M}$ denotes the epansion of the image of operator $\mathcal{C}_{M}$ to pure quaternion. 


\section{Governing equations of the beam}

The complete set of the beam equations consists of the kinematic equations (40)-(41), the constitutive equations (46)-(47), and the equilibrium equations (42)-(43). In the quaternion representation, this set of equations reads:

$$
\begin{aligned}
& \boldsymbol{f}_{1}=\boldsymbol{N}_{g}^{\prime}(x)+\boldsymbol{n}_{g}(x)=\mathbf{0} \\
& \boldsymbol{f}_{2}=\widehat{\boldsymbol{M}}_{g}^{\prime}(x)+\widehat{\boldsymbol{m}}_{g}(x)-\boldsymbol{N}_{g}\left(\widehat{x) \times \boldsymbol{r}_{g}^{\prime}}(x)=\widehat{\mathbf{0}}\right. \\
& \boldsymbol{f}_{3}=\left[\widehat{\boldsymbol{q}}(x) \circ \widehat{\mathcal{C}}_{N}\left(\boldsymbol{\gamma}_{G}(x), \boldsymbol{\kappa}_{G}(x)\right) \circ \widehat{\boldsymbol{q}}^{*}(x)\right]_{\mathbb{R}^{3}}-\boldsymbol{N}_{g}(x)=\mathbf{0} \\
& \boldsymbol{f}_{4}=\widehat{\boldsymbol{q}}(x) \circ \widehat{\mathcal{C}}_{M}\left(\boldsymbol{\gamma}_{G}(x), \boldsymbol{\kappa}_{G}(x)\right) \circ \widehat{\boldsymbol{q}}^{*}(x)-\widehat{\boldsymbol{M}}_{g}(x)=\widehat{\mathbf{0}} \\
& \boldsymbol{f}_{5}=\left[\widehat{\boldsymbol{q}}^{*}(x) \circ \widehat{\boldsymbol{r}}_{g}^{\prime}(x) \circ \widehat{\boldsymbol{q}}(x)\right]_{R^{3}}-\left(\gamma_{G}(x)-\boldsymbol{c}_{G}(x)\right)=\mathbf{0} \\
& \boldsymbol{f}_{6}=2 \widehat{\boldsymbol{q}}^{*}(x) \circ \widehat{\boldsymbol{q}}^{\prime}(x)-\left(\widehat{\boldsymbol{\kappa}}_{G}(x)-\widehat{\boldsymbol{d}}_{G}(x)\right)=\widehat{\mathbf{0}} .
\end{aligned}
$$

In equations (48)-(53), the hat over a symbol $\widehat{\bullet}$ designates a quaternion or an expansion of a vector to a pure quaternion, while the brackets [ $]_{\mathbb{R}^{3}}$ designate the vector part of a quaternion. It must be emphasized that equations (49), (51) and (53) related to moments and rotational strains are considered in four dimensions. This enables that the four components of the rotational quaternion are taken as independent scalar variables. The remaining equations are restricted to stay in three dimensions to avoid possible numerical singularities at the scalar part of the quaternions. The boundary conditions at the two boundaries, $x=0$ and $x=L$, are

$$
\begin{aligned}
& \boldsymbol{h}_{1}=\boldsymbol{F}_{g}^{0}+\boldsymbol{N}_{g}(0)=\mathbf{0} \\
& \boldsymbol{h}_{2}=\boldsymbol{P}_{g}^{0}+\boldsymbol{M}_{g}(0)=\mathbf{0} \\
& \boldsymbol{h}_{3}=\boldsymbol{F}_{g}^{L}-\boldsymbol{N}_{g}(L)=\mathbf{0} \\
& \boldsymbol{h}_{4}=\boldsymbol{P}_{g}^{L}-\boldsymbol{M}_{g}(L)=\mathbf{0} .
\end{aligned}
$$

$\boldsymbol{F}_{g}^{0}, \boldsymbol{P}_{g}^{0}, \boldsymbol{F}_{g}^{L}, \boldsymbol{P}_{g}^{L}$ are the external point forces and moments at the two boundary points.

The solution of the boundary value problem (48)-(57) is achieved in several successive steps. The fist step consists of integrating equations (42)-(43) for $\boldsymbol{N}_{g}$ and $\boldsymbol{M}_{g}$ assuming that $\boldsymbol{n}_{g}$ and $\boldsymbol{m}_{g}$ are given analytic functions of $x$ :

$$
\begin{aligned}
& \boldsymbol{N}_{g}(x)=\boldsymbol{N}_{g}\left(\frac{L}{2}\right)+\int_{x}^{L / 2} \boldsymbol{n}_{g}(\xi) d \xi \\
& \boldsymbol{M}_{g}(x)=\boldsymbol{M}_{g}\left(\frac{L}{2}\right)+\int_{x}^{L / 2} \boldsymbol{m}_{g}(\xi) d \xi-\int_{x}^{L / 2} \boldsymbol{N}_{g}(\xi) \times \boldsymbol{r}_{g}^{\prime}(\xi) d \xi
\end{aligned}
$$

Note that the integrations in equations (58) and (59) are performed with respect to the midspan of the beam to preserve the symmetry of the solution with respect to the geometrical symmetry point of the beam. 
In the next step, we insert $\boldsymbol{\gamma}_{G}$ and $\widehat{\boldsymbol{\kappa}}_{G}$ from (52)-(53), and $\boldsymbol{N}_{g}$ and $\widehat{\boldsymbol{M}}_{g}$ from (48)-(49) into the weak (differentiated) form of equations (50)-(51). After considering (58) we obtain the following result:

$$
\begin{aligned}
& \mathfrak{f}_{1}=\boldsymbol{f}_{3}^{\prime}=\left[\widehat{\boldsymbol{q}}(x) \circ \widehat{\mathcal{C}}_{N}\left(\boldsymbol{\gamma}_{G}(x), \boldsymbol{\kappa}_{G}(x)\right) \circ \widehat{\boldsymbol{q}}^{*}(x)\right]_{\mathbb{R}^{3}}^{\prime}+\boldsymbol{n}_{g}(x)=\mathbf{0} \\
& \mathfrak{f}_{2}=\boldsymbol{f}_{4}^{\prime}=\left(\widehat{\boldsymbol{q}}(x) \circ \widehat{\mathcal{C}}_{M}\left(\boldsymbol{\gamma}_{G}(x), \boldsymbol{\kappa}_{G}(x)\right) \circ \widehat{\boldsymbol{q}}^{*}(x)\right)^{\prime} \\
& +\widehat{\boldsymbol{m}}_{g}(x)-\boldsymbol{N}_{g}\left(\widehat{L / 2) \times \boldsymbol{r}_{g}^{\prime}}(x)-\int_{x}^{L / 2} \boldsymbol{n}_{g}(\xi) \widehat{d \xi \times \boldsymbol{r}_{g}^{\prime}}(x)=\widehat{\mathbf{0}},\right.
\end{aligned}
$$

where, from (52)-(53),

$$
\begin{aligned}
& \boldsymbol{\gamma}_{G}(x)=\left[\widehat{\boldsymbol{q}}^{*}(x) \circ \widehat{\boldsymbol{r}}_{g}^{\prime}(x) \circ \widehat{\boldsymbol{q}}(x)\right]_{\mathbb{R}^{3}}+\boldsymbol{c}_{G}(x) \\
& \boldsymbol{\kappa}_{G}(x)=2\left[\widehat{\boldsymbol{q}}^{*}(x) \circ \widehat{\boldsymbol{q}}^{\prime}(x)\right]_{\mathbb{R}^{3}}+\boldsymbol{d}_{G}(x) .
\end{aligned}
$$

The integrated forms of the equilibrium equations (58) and (59) are also taken into account when rewriting the boundary conditions as

$$
\begin{aligned}
& \boldsymbol{h}_{1}=\boldsymbol{F}_{g}^{0}+\boldsymbol{N}_{g}\left(\frac{L}{2}\right)+\int_{0}^{L / 2} \boldsymbol{n}_{g}(\xi) d \xi=\mathbf{0} \\
& \boldsymbol{h}_{2}=\boldsymbol{P}_{g}^{0}+\boldsymbol{M}_{g}\left(\frac{L}{2}\right)+\int_{0}^{L / 2} \boldsymbol{m}_{g}(\xi) d \xi-\int_{0}^{L / 2} \boldsymbol{N}_{g}(\xi) \times \boldsymbol{r}_{g}^{\prime}(\xi) d \xi=\mathbf{0} \\
& \boldsymbol{h}_{3}=\boldsymbol{F}_{g}^{L}-\boldsymbol{N}_{g}\left(\frac{L}{2}\right)-\int_{L}^{L / 2} \boldsymbol{n}_{g}(\xi) d \xi=\mathbf{0} \\
& \boldsymbol{h}_{4}=\boldsymbol{P}_{g}^{L}-\boldsymbol{M}_{g}\left(\frac{L}{2}\right)-\int_{L}^{L / 2} \boldsymbol{m}_{g}(\xi) d \xi+\int_{L}^{L / 2} \boldsymbol{N}_{g}(\xi) \times \boldsymbol{r}_{g}^{\prime}(\xi) d \xi=\mathbf{0} .
\end{aligned}
$$

After the last terms in equations (65) and (67) are integrated by parts and the result rearranged considering (58) we get

$$
\begin{aligned}
\boldsymbol{h}_{1}= & \boldsymbol{F}_{g}^{0}+\boldsymbol{N}_{g}^{L / 2}+\int_{0}^{L / 2} \boldsymbol{n}_{g} d x=\mathbf{0} \\
\boldsymbol{h}_{2}= & \boldsymbol{P}_{g}^{0}+\boldsymbol{M}_{g}^{L / 2}-\boldsymbol{N}_{g}^{L / 2} \times\left(\boldsymbol{r}_{g}^{L / 2}-\boldsymbol{r}_{g}^{0}\right) \\
& \quad-\int_{0}^{L / 2} \boldsymbol{n}_{g} \times\left(\boldsymbol{r}_{g}-\boldsymbol{r}_{g}^{0}\right) d x+\int_{0}^{L / 2} \boldsymbol{m}_{g} d x=\mathbf{0} \\
\boldsymbol{h}_{3}= & \boldsymbol{F}_{g}^{L}-\boldsymbol{N}_{g}^{L / 2}+\int_{L / 2}^{L} \boldsymbol{n}_{g} d x=\mathbf{0} \\
\boldsymbol{h}_{4}= & \boldsymbol{P}_{g}^{L}-\boldsymbol{M}_{g}^{L / 2}-\boldsymbol{N}_{g}^{L / 2} \times\left(\boldsymbol{r}_{g}^{L}-\boldsymbol{r}_{g}^{L / 2}\right) \\
& \quad+\int_{L / 2}^{L} \boldsymbol{n}_{g} \times\left(\boldsymbol{r}_{g}^{L}-\boldsymbol{r}_{g}\right) d x+\int_{L / 2}^{L} \boldsymbol{m}_{g} d x=\mathbf{0} .
\end{aligned}
$$

The values of stress resultants $\boldsymbol{N}_{g}$ and $\boldsymbol{M}_{g}$ at the midspan of the beam element, $x=L / 2$, are obtained from the consistency equations, when applied at 
$x=L / 2$ :

$$
\begin{aligned}
\boldsymbol{N}_{g}^{L / 2} & =\left[\widehat{\boldsymbol{q}}^{L / 2} \circ \widehat{\mathcal{C}}_{N}\left(\boldsymbol{\gamma}_{G}^{L / 2}, \boldsymbol{\kappa}_{G}^{L / 2}\right) \circ \widehat{\boldsymbol{q}}^{* L / 2}\right]_{\mathbb{R}^{3}} \\
\boldsymbol{M}_{g}^{L / 2} & =\left[\widehat{\boldsymbol{q}}^{L / 2} \circ \widehat{\mathcal{C}}_{M}\left(\boldsymbol{\gamma}_{G}^{L / 2}, \boldsymbol{\kappa}_{G}^{L / 2}\right) \circ \widehat{\boldsymbol{q}}^{* L / 2}\right]_{\mathbb{R}^{3}} .
\end{aligned}
$$

Equations (60)-(61) and (68)-(71) along with the auxiliary relations (62)-(63) and (72)-(73) represent the complete set of the governing equations of the present beam formulation. The unknowns in these equations are the position vector of the deformed line of centroids, $\boldsymbol{r}_{g}(x)$, and the rotational quaternion, $\widehat{\boldsymbol{q}}(x)$, describing the current rotation of the cross-sections.

\section{Numerical solution}

\subsection{Discrete governing equations}

In the numerical formulation, functions $\boldsymbol{r}_{g}(x)$ and $\widehat{\boldsymbol{q}}(x)$ are replaced by a set of their unknown values $\boldsymbol{r}_{g}^{p}$ and $\widehat{\boldsymbol{q}}^{p}$ at $N+2$ discrete points $x_{p} ; p=0$, $\ldots, N+1$, chosen from the interval $[0, L]$ so that $x_{0}=0$ and $x_{N+1}=L$. Equations (60)-(61) are required to be satisfied at $N$ pre-selected collocation points $y_{k} ; k=1, \ldots, N$. For simplicity, they are here taken to coincide with internal points $x_{p} ; p=1, \ldots, N$. After such a discretization is made, a set of $2 N+4$ matrix equations is obtained, which needs to be solved for $2 N+4$ unknowns $\boldsymbol{r}_{g}^{p}$ and $\widehat{\boldsymbol{q}}^{p}$ :

$$
\begin{aligned}
& \mathfrak{f}_{1}^{k}=\left[\widehat{\boldsymbol{q}}^{k} \circ \widehat{\mathcal{C}}_{N}\left(\boldsymbol{\gamma}_{G}^{k}, \boldsymbol{\kappa}_{G}^{k}\right) \circ \widehat{\boldsymbol{q}}^{* k}\right]_{\mathbb{R}^{3}}^{\prime}+\boldsymbol{n}_{g}^{k}=\mathbf{0} \\
& \mathfrak{f}_{2}^{k}=\left(\widehat{\boldsymbol{q}}^{k} \circ \widehat{\mathcal{C}}_{M}\left(\boldsymbol{\gamma}_{G}^{k}, \boldsymbol{\kappa}_{G}^{k}\right) \circ \widehat{\boldsymbol{q}}^{* k}\right)^{\prime} \\
& +\widehat{\boldsymbol{m}}_{g}^{k}-\boldsymbol{N}_{g}^{\widehat{L / 2} \times \boldsymbol{r}_{g}^{\prime k}}+\int_{x_{k}}^{L / 2} \boldsymbol{n}_{g} \widehat{d x \times \boldsymbol{r}_{g}^{\prime k}}=\widehat{\mathbf{0}} \\
& \boldsymbol{h}_{1}=\boldsymbol{F}_{g}^{0}+\boldsymbol{N}_{g}^{L / 2}+\int_{0}^{L / 2} \boldsymbol{n}_{g} d x=\mathbf{0} \\
& \boldsymbol{h}_{2}=\boldsymbol{P}_{g}^{0}+\boldsymbol{M}_{g}^{L / 2}-\boldsymbol{N}_{g}^{L / 2} \times\left(\boldsymbol{r}_{g}^{L / 2}-\boldsymbol{r}_{g}^{0}\right) \\
& -\int_{0}^{L / 2} \boldsymbol{n}_{g} \times\left(\boldsymbol{r}_{g}-\boldsymbol{r}_{g}^{0}\right) d x+\int_{0}^{L / 2} \boldsymbol{m}_{g} d x=\mathbf{0} \\
& \boldsymbol{h}_{3}=\boldsymbol{F}_{g}^{L}-\boldsymbol{N}_{g}^{L / 2}+\int_{L / 2}^{L} \boldsymbol{n}_{g} d x=\mathbf{0} \\
& \boldsymbol{h}_{4}=\boldsymbol{P}_{g}^{L}-\boldsymbol{M}_{g}^{L / 2}-\boldsymbol{N}_{g}^{L / 2} \times\left(\boldsymbol{r}_{g}^{L}-\boldsymbol{r}_{g}^{L / 2}\right) \\
& +\int_{L / 2}^{L} \boldsymbol{n}_{g} \times\left(\boldsymbol{r}_{g}^{L}-\boldsymbol{r}_{g}\right) d x+\int_{L / 2}^{L} \boldsymbol{m}_{g} d x=\mathbf{0} .
\end{aligned}
$$


The system of non-linear algebraic equations (74)-(79) is solved by Newton's method. This requires the linearization of algebraic equations to obtain a system of linear equations, which is solved iteratively

$$
\mathbf{K}^{[n]} \delta \boldsymbol{y}=-\boldsymbol{f}^{[n]}, \quad \text { for } n=0,1,2, \ldots
$$

until the accuracy is sufficient. Here $\mathbf{K}^{[n]}$ is the global tangent stiffness matrix, $\boldsymbol{f}^{[n]}$ the residual vector, and $\delta \boldsymbol{y}$ a vector of corrections of unknowns.

\subsection{Linearization of governing equations}

In discussing the linearization, we have to consider that the variations of the primary unknowns are arbitrary independent one-column matrices, denoted by $\delta \boldsymbol{r}_{g}^{p}$ and $\delta \widehat{\boldsymbol{q}}^{p}$. Next, the governing equations depend also on the derivatives of the primary unknowns with respect to $x$. That is why we have to introduce an interpolation of the variations along the element here chosen in the form

$$
\begin{aligned}
\delta \boldsymbol{r}_{g}(x) & =\sum_{p=0}^{N+1} L_{p}(x) \delta \boldsymbol{r}_{g}^{p} \\
\delta \widehat{\boldsymbol{q}}(x) & =\sum_{p=0}^{N+1} L_{p}(x) \delta \widehat{\boldsymbol{q}}^{p},
\end{aligned}
$$

where $L_{p}(x)$ denotes at least twice differentiable interpolation functions which is due to the weak form of internal equilibrium equations employed in our formulation. The first and the second derivatives at any point $x$ are then obtained as

$$
\begin{array}{rlrl}
\delta \boldsymbol{r}_{g}^{\prime} & =\sum_{p=0}^{N+1} L_{p}^{\prime}(x) \delta \boldsymbol{r}_{g}^{p} & \delta \boldsymbol{r}_{g}^{\prime \prime} & =\sum_{p=0}^{N+1} L_{p}^{\prime \prime}(x) \delta \boldsymbol{r}_{g}^{p} \\
\delta \widehat{\boldsymbol{q}}^{\prime}=\sum_{p=0}^{N+1} L_{p}^{\prime}(x) \delta \widehat{\boldsymbol{q}}^{p} & \delta \widehat{\boldsymbol{q}}^{\prime \prime} & =\sum_{p=0}^{N+1} L_{p}^{\prime \prime}(x) \delta \widehat{\boldsymbol{q}}^{p} .
\end{array}
$$

The deduction of the linearization of equations (74)-(79) is greatly simplified, if variations of the quantities involved are prepared in advance. The linearization of strains with respect to primary unknowns has already been discussed and need not be repeated, i.e. (see equation (37))

$$
\delta \widehat{\boldsymbol{\kappa}}_{G}=2 \delta \widehat{\boldsymbol{q}}^{*} \circ \widehat{\boldsymbol{q}}^{\prime}+2 \widehat{\boldsymbol{q}}^{*} \circ \delta \widehat{\boldsymbol{q}}^{\prime} .
$$

Linearizing the definition of strain vector $\gamma_{G}$ in equation (40) gives

$$
\delta \boldsymbol{\gamma}_{G}=\left[\delta \widehat{\boldsymbol{q}}^{*} \circ \widehat{\boldsymbol{r}}_{g}^{\prime} \circ \widehat{\boldsymbol{q}}+\widehat{\boldsymbol{q}}^{*} \circ \delta \widehat{\boldsymbol{r}}_{g}^{\prime} \circ \widehat{\boldsymbol{q}}+\widehat{\boldsymbol{q}}^{*} \circ \widehat{\boldsymbol{r}}_{g}^{\prime} \circ \delta \widehat{\boldsymbol{q}}\right]_{\mathbb{R}^{3}} .
$$


The linearization of the derivatives of strain measures follows from (85) and (86):

$$
\begin{aligned}
\delta \boldsymbol{\gamma}_{G}^{\prime} & =-\left[\delta \widehat{\boldsymbol{q}}^{* \prime} \circ \widehat{\boldsymbol{r}}_{g}^{\prime} \circ \widehat{\boldsymbol{q}}+\delta \widehat{\boldsymbol{q}}^{*} \circ \widehat{\boldsymbol{r}}_{g}^{\prime \prime} \circ \widehat{\boldsymbol{q}}+\delta \widehat{\boldsymbol{q}}^{*} \circ \widehat{\boldsymbol{r}}_{g}^{\prime} \circ \widehat{\boldsymbol{q}}^{\prime}+\widehat{\boldsymbol{q}}^{* \prime} \circ \delta \widehat{\boldsymbol{r}}_{g}^{\prime} \circ \widehat{\boldsymbol{q}}+\widehat{\boldsymbol{q}}^{*} \circ \delta \widehat{\boldsymbol{r}}_{g}^{\prime \prime} \circ \widehat{\boldsymbol{q}}\right. \\
& \left.+\widehat{\boldsymbol{q}}^{*} \circ \delta \widehat{\boldsymbol{r}}_{g}^{\prime} \circ \widehat{\boldsymbol{q}}^{\prime}+\widehat{\boldsymbol{q}}^{* \prime} \circ \widehat{\boldsymbol{r}}_{g}^{\prime} \circ \delta \widehat{\boldsymbol{q}}+\widehat{\boldsymbol{q}}^{*} \circ \widehat{\boldsymbol{r}}_{g}^{\prime \prime} \circ \delta \widehat{\boldsymbol{q}}^{\prime}+\widehat{\boldsymbol{q}}^{*} \circ \widehat{\boldsymbol{r}}_{g}^{\prime} \circ \delta \widehat{\boldsymbol{q}}^{\prime}\right]_{\mathbb{R}^{3}} \\
\delta \widehat{\boldsymbol{\kappa}}_{G}^{\prime} & =2 \delta \widehat{\boldsymbol{q}}^{* \prime} \circ \widehat{\boldsymbol{q}}^{\prime}+2 \delta \widehat{\boldsymbol{q}}^{*} \circ \widehat{\boldsymbol{q}}^{\prime \prime}+2 \widehat{\boldsymbol{q}}^{* \prime} \circ \delta \widehat{\boldsymbol{q}}^{\prime}+2 \widehat{\boldsymbol{q}}^{*} \circ \delta \widehat{\boldsymbol{q}}^{\prime \prime}
\end{aligned}
$$

In varying operators $\mathcal{C}_{N}$ and $\mathcal{C}_{M}$, we have to consider that they are dependent only on $\boldsymbol{\gamma}_{G}$ and $\boldsymbol{\kappa}_{G}$. Hence

$$
\begin{aligned}
\delta \mathcal{C}_{N} & =\mathbf{C}_{\gamma \gamma} \delta \boldsymbol{\gamma}_{G}+\mathbf{C}_{\gamma \kappa} \delta \boldsymbol{\kappa}_{G} \\
\delta \mathcal{C}_{M} & =\mathbf{C}_{\kappa \gamma} \delta \boldsymbol{\gamma}_{G}+\mathbf{C}_{\kappa \kappa} \delta \boldsymbol{\kappa}_{G} \\
\delta \mathcal{C}_{N}^{\prime} & =\mathbf{C}_{\gamma \gamma}^{\prime} \delta \boldsymbol{\gamma}_{G}+\mathbf{C}_{\gamma \kappa}^{\prime} \delta \boldsymbol{\kappa}_{G}+\mathbf{C}_{\gamma \gamma} \delta \boldsymbol{\gamma}_{G}^{\prime}+\mathbf{C}_{\gamma \kappa} \delta \boldsymbol{\kappa}_{G}^{\prime} \\
\delta \mathcal{C}_{M}^{\prime} & =\mathbf{C}_{\kappa \gamma}^{\prime} \delta \boldsymbol{\gamma}_{G}+\mathbf{C}_{\kappa \kappa}^{\prime} \delta \boldsymbol{\kappa}_{G}+\mathbf{C}_{\kappa \gamma} \delta \boldsymbol{\gamma}_{G}^{\prime}+\mathbf{C}_{\kappa \kappa} \delta \boldsymbol{\kappa}_{G}^{\prime},
\end{aligned}
$$

where $\mathbf{C}_{\gamma \gamma}, \mathbf{C}_{\gamma \kappa}, \mathbf{C}_{\kappa \gamma}, \mathbf{C}_{\kappa \kappa}$ are the sub-matrices of the cross-section constitutive tangent matrix defined as

$$
\begin{aligned}
\mathbf{C}_{\gamma \gamma}=\left[\frac{\partial \mathcal{C}_{N}^{i}}{\partial \gamma_{j}}\right]_{i, j=1,2,3} & \mathbf{C}_{\gamma \kappa}=\left[\frac{\partial \mathcal{C}_{N}^{i}}{\partial \kappa_{j}}\right]_{i, j=1,2,3} \\
\mathbf{C}_{\kappa \gamma}=\left[\frac{\partial \mathcal{C}_{M}^{i}}{\partial \gamma_{j}}\right]_{i, j=1,2,3} & \mathbf{C}_{\kappa \kappa}=\left[\frac{\partial \mathcal{C}_{M}^{i}}{\partial \kappa_{j}}\right]_{i, j=1,2,3} .
\end{aligned}
$$

After these preparations have been done, the linear form of equations (74)-(79) is easily derived and is thus not presented here. Prior to solving the system of linear equations (80), the quaternion form of linearized equations should be rearranged into the matrix form using rules (11)-(12).

\subsection{Update procedure}

As a result of an iteration step, the corrections $\delta \boldsymbol{r}_{g}^{p}$ and $\delta \widehat{\boldsymbol{q}}^{p}$ are obtained. In application of Newton's method in linear vector spaces, the correction is added to the previous solution vector: $\boldsymbol{y}^{[n+1]}=\boldsymbol{y}^{[n]}+\delta \boldsymbol{y}$. This is, however, not the case in the non-linear spaces which requires a non-additive update. As the position vectors are linear quantities, their corrections are added to the current iterative values:

$$
\boldsymbol{r}_{g}^{p[n+1]}=\boldsymbol{r}_{g}^{p[n]}+\delta \boldsymbol{r}_{g}^{p}
$$

In updating the rotational quaternions, we must consider their multiplicative nature. A new, improved value of the rotational quaternion is obtained by mapping from the tangent space onto the configuration space of rotational quaternions. This may be achieved by using equations (30)-(31). Note that 
$\delta \widehat{\boldsymbol{q}}^{p}$ is a quaternion with a non-unit norm; its normalization would resolve this problem, but such a reduced quaternion would be inconsistent with the theory of three-dimensional rotations and their variations. To obtain the incremental rotational quaternion in a consistent manner, the following formula directly derived from (30)-(31) is used

$$
\Delta \widehat{\boldsymbol{q}}^{p}=\cos \left|\delta \widehat{\boldsymbol{q}}^{p} \circ \widehat{\boldsymbol{q}}^{* p}\right|+\frac{\left[\delta \widehat{\boldsymbol{q}}^{p} \circ \widehat{\boldsymbol{q}}^{* p}\right]_{\mathbb{R}^{3}}}{\left|\delta \widehat{\boldsymbol{q}}^{p} \circ \widehat{\boldsymbol{q}}^{* p}\right|} \sin \left|\delta \widehat{\boldsymbol{q}}^{p} \circ \widehat{\boldsymbol{q}}^{* p}\right| .
$$

The improved rotational quaternion is then obtained by the left quaternion multiplication as

$$
\widehat{\boldsymbol{q}}^{p[n+1]}=\Delta \widehat{\boldsymbol{q}}^{p} \circ \widehat{\boldsymbol{q}}^{p[n]} .
$$

A similar result is reported by Lasenby and McRobbie [18] for the update procedure in the Clifford algebra.

The solution of non-linear equations (74)-(79) requires the first and the second derivatives of the primary unknowns to be given at the chosen discrete points. This does not represent a problem for the position vectors, where the same type of interpolation as the one for variations can be used. Hence we have

$$
\begin{aligned}
\boldsymbol{r}_{g}^{\prime}(x) & =\sum_{p=0}^{N+1} L_{p}^{\prime}(x) \boldsymbol{r}_{g}^{p} \\
\boldsymbol{r}_{g}^{\prime \prime}(x) & =\sum_{p=0}^{N+1} L_{p}^{\prime \prime}(x) \boldsymbol{r}_{g}^{p}
\end{aligned}
$$

This type of interpolation is not permitted for the approximation of quaternions due to their multiplicative nature. The right way to obtain the derivatives of the rotational quaternion is to use equation (28) along with a well known property on the additivity of the rotational strains, see, e.g. [34, page 5229]. Prior to obtain the derivative of the rotational quaternion from equation (28), we must compute the current rotational quaternion and the current rotational strain. The current rotational strain is obtained by inserting (91) into $(28)$

$$
\begin{aligned}
\widehat{\boldsymbol{\kappa}}_{g}^{[n+1]} & =2\left(\Delta \widehat{\boldsymbol{q}} \circ \widehat{\boldsymbol{q}}^{[n]}\right)^{\prime} \circ \widehat{\boldsymbol{q}}^{*[n]} \circ \Delta \widehat{\boldsymbol{q}}^{*} \\
& =2 \Delta \widehat{\boldsymbol{q}}^{\prime} \circ \Delta \widehat{\boldsymbol{q}}^{*}+2 \Delta \widehat{\boldsymbol{q}} \circ \widehat{\boldsymbol{q}}^{[n] \prime} \circ \widehat{\boldsymbol{q}}^{*[n]} \circ \Delta \widehat{\boldsymbol{q}}^{*} \\
& =\Delta \widehat{\boldsymbol{\kappa}}_{g}+\Delta \widehat{\boldsymbol{q}} \circ \widehat{\boldsymbol{\kappa}}_{g}^{[n]} \circ \Delta \widehat{\boldsymbol{q}}^{*} .
\end{aligned}
$$

The transformation into the local basis $\left(G^{[n+1]}\right)$ gives

$$
\begin{aligned}
\widehat{\boldsymbol{\kappa}}_{G[n+1]}^{[n+1]} & =\widehat{\boldsymbol{q}}^{*[n+1]} \circ \Delta \widehat{\boldsymbol{\kappa}}_{g} \circ \widehat{\boldsymbol{q}}^{[n+1]}+\widehat{\boldsymbol{q}}^{*[n+1]} \circ \Delta \widehat{\boldsymbol{q}} \circ \widehat{\boldsymbol{\kappa}}_{g}^{[n]} \circ \Delta \widehat{\boldsymbol{q}}^{*} \circ \widehat{\boldsymbol{q}}^{[n+1]} \\
& =\Delta \widehat{\boldsymbol{\kappa}}_{G^{[n+1]}}+\widehat{\boldsymbol{\kappa}}_{G^{[n]}}^{[n]} .
\end{aligned}
$$


The result is significant: the sum of the curvature quaternion with respect to the local basis in iteration $n$, and its correction with respect to the local basis in iteration $n+1$, gives the curvature in iteration $n+1$ with respect to the local basis in current iteration $n+1$. Inserting (82) into (90) gives the incremental rotational quaternion at an arbitrary point $x$

$$
\begin{aligned}
\Delta \widehat{\boldsymbol{q}}(x)=\cos \mid & \left|\sum_{p=0}^{N+1} L_{p}(x) \delta \widehat{\boldsymbol{q}}^{p} \circ \widehat{\boldsymbol{q}}^{*}(x)\right| \\
& +\frac{\left[\sum_{p=0}^{N+1} L_{p}(x) \delta \widehat{\boldsymbol{q}}^{p} \circ \widehat{\boldsymbol{q}}^{*}(x)\right]_{\mathbb{R}^{3}}}{\left|\sum_{p=0}^{N+1} L_{p}(x) \delta \widehat{\boldsymbol{q}}^{p} \circ \widehat{\boldsymbol{q}}^{*}(x)\right|} \sin \left|\sum_{p=0}^{N+1} L_{p}(x) \delta \widehat{\boldsymbol{q}}^{p} \circ \widehat{\boldsymbol{q}}^{*}(x)\right| .
\end{aligned}
$$

Inserting (96) in (91) gives the rotational quaternion in iteration $n+1$, and applying (96) in (95) yields the curvature quaternion in iteration $n+1$. After the rotational and the curvature quaternions in iteration $n+1$ have been obtained, $\widehat{\boldsymbol{q}}^{[n+1] \prime}$ is evaluated from equation (28).

To obtain the second derivative of the rotational quaternion, it is convenient to differentiate equation (94) with respect to $x$

$$
\widehat{\boldsymbol{\kappa}}_{g}^{[n+1] \prime}=\Delta \widehat{\boldsymbol{\kappa}}_{g}^{\prime}+\Delta \widehat{\boldsymbol{q}}^{\prime} \circ \widehat{\boldsymbol{\kappa}}_{g}^{[n]} \circ \Delta \widehat{\boldsymbol{q}}^{*}+\Delta \widehat{\boldsymbol{q}} \circ \widehat{\boldsymbol{\kappa}}_{g}^{[n] \prime} \circ \Delta \widehat{\boldsymbol{q}}^{*}+\Delta \widehat{\boldsymbol{q}} \circ \widehat{\boldsymbol{\kappa}}_{g}^{[n]} \circ \Delta \widehat{\boldsymbol{q}}^{* \prime}
$$

and transform it into the local basis at $n+1\left(G^{[n+1]}\right)$ :

$$
\begin{aligned}
\widehat{\boldsymbol{\kappa}}_{G}^{[n+1] \prime}= & \widehat{\boldsymbol{q}}^{*[n+1]} \circ \Delta \widehat{\boldsymbol{\kappa}}_{g}^{\prime} \circ \widehat{\boldsymbol{q}}^{[n+1]}+\widehat{\boldsymbol{q}}^{*[n+1]} \circ \Delta \widehat{\boldsymbol{q}}^{\prime} \circ \widehat{\boldsymbol{\kappa}}_{g}^{[n]} \circ \widehat{\boldsymbol{q}}^{[n]}+\widehat{\boldsymbol{q}}^{*[n]} \circ \widehat{\boldsymbol{\kappa}}_{g}^{[n] \prime} \circ \widehat{\boldsymbol{q}}^{[n]} \\
& \quad+\widehat{\boldsymbol{q}}^{*[n]} \circ \widehat{\boldsymbol{\kappa}}_{g}^{[n]} \circ \Delta \widehat{\boldsymbol{q}}^{* \prime} \circ \widehat{\boldsymbol{q}}^{[n+1]} \\
= & \Delta \widehat{\boldsymbol{\kappa}}_{G^{[n+1]}}^{\prime}+\frac{1}{2} \widehat{\boldsymbol{q}}^{*[n+1]} \circ \Delta \widehat{\boldsymbol{\kappa}}_{g} \circ \Delta \widehat{\boldsymbol{q}} \circ \widehat{\boldsymbol{\kappa}}_{g}^{[n]} \circ \widehat{\boldsymbol{q}}^{[n]} \\
& \quad+\widehat{\boldsymbol{\kappa}}_{G[n]}^{[n] \prime}+\frac{1}{2} \widehat{\boldsymbol{q}}^{*[n]} \circ \widehat{\boldsymbol{\kappa}}_{g}^{[n]} \circ \Delta \widehat{\boldsymbol{q}}^{*} \circ \Delta \widehat{\boldsymbol{\kappa}}_{g}^{*} \circ \widehat{\boldsymbol{q}}^{[n+1]} \\
= & \widehat{\boldsymbol{\kappa}}_{G^{[n]}}^{[n] \prime}+\Delta \widehat{\boldsymbol{\kappa}}_{G^{[n+1]}}^{\prime}+\frac{1}{2} \Delta \widehat{\boldsymbol{\kappa}}_{G^{[n+1]}} \circ \widehat{\boldsymbol{\kappa}}_{G}^{[n]}+\frac{1}{2} \widehat{\boldsymbol{\kappa}}_{G^{[n]}}^{[n]} \circ \Delta \widehat{\boldsymbol{\kappa}}_{G^{[n+1]}}^{* n+1} \\
= & \widehat{\boldsymbol{\kappa}}_{G^{[n]}}^{[n] \prime}+\Delta \widehat{\boldsymbol{\kappa}}_{G^{[n+1]}}^{\prime}+\frac{1}{2}\left(\Delta \widehat{\boldsymbol{\kappa}}_{G^{[n+1]}} \circ \widehat{\boldsymbol{\kappa}}_{G^{[n]}}^{[n]}-\frac{1}{2} \widehat{\boldsymbol{\kappa}}_{G[n]}^{[n]} \circ \Delta \widehat{\boldsymbol{\kappa}}_{G^{[n+1]}}\right) .
\end{aligned}
$$

Once the first derivative of the rotational strain is known, the second derivative of the total rotational vector is extracted from the differentiated equation (28).

\section{$7 \quad$ Numerical examples}

We present several classical numerical examples in order to demonstrate the validity, performance and the accuracy of the proposed approach. Arbitrary finite-size initial bending and/or twisting strains along the beam can be prescribed at the unloaded configuration, and the model is capable of considering 
finite displacements, rotations and strains. Numerical solutions were obtained in the Matlab [17] computing environment. Here we limit ourselves to linear elastic materials whose linear operators $\mathcal{C}_{N}$ and $\mathcal{C}_{M}$ in (48)-(49) are diagonal and written as:

$$
\begin{aligned}
\mathcal{C}_{N} & =\left[\begin{array}{ccc}
E A_{1} & 0 & 0 \\
0 & G A_{2} & 0 \\
0 & 0 & G A_{3}
\end{array}\right] \boldsymbol{\gamma}_{G}=\mathbf{C}_{N} \boldsymbol{\gamma}_{G}, \\
\mathcal{C}_{M} & =\left[\begin{array}{ccc}
G J_{1} & 0 & 0 \\
0 & E J_{2} & 0 \\
0 & 0 & E J_{3}
\end{array}\right] \boldsymbol{\kappa}_{G}=\mathbf{C}_{M} \boldsymbol{\kappa}_{G} .
\end{aligned}
$$

$E$ and $G$ denote elastic and shear moduli of material; $A_{1}$ is the cross-sectional area; $J_{1}$ is the torsional inertial moment of the cross-section; $A_{2}$ and $A_{3}$ are the effective shear areas in the principal inertial directions $\vec{G}_{2}$ and $\vec{G}_{3}$ of the crosssection; $J_{2}$ and $J_{3}$ are the corresponding principal bending inertial moments of the cross-section.

The accuracy of the present numerical model can be enhanced by (i) the increase of the number of elements, $n_{e}$, and/or (ii) by the increase of the number of discretization points within the element, $N$. Several combinations will be considered to show their effects.

\subsection{Illustration of insensibility to shear locking}

To demonstrate that the present formulation overcomes the shear locking problem, we study a straight cantilever beam under a vertical tip load $F=1$ (Figure 2). The geometric and material properties of the cantilever are:

$$
E=10^{7} \quad G=10^{13} \quad L=1 \quad t=0.1 .
$$

Note that the shear modulus $G$ was taken exceedingly large as the problem of shear locking becomes severe by increasing $G$. On the other hand, in conventional displacement-based finite elements, locking progresses rapidly as the height of element decreases. The height $h$ of the beam was therefore varied from 0.1 (small) to 10 (large). Our numerical results for the tip displacement $u_{Z}$ are compared with the exact solution $u_{Z \text {,ref }}$. The variation of the error $u_{Z} / u_{Z \text {,ref }}$ is shown with respect to the structural parameter $G L^{2} / E h^{2}$ in logarithmic scale in Figure 3. 


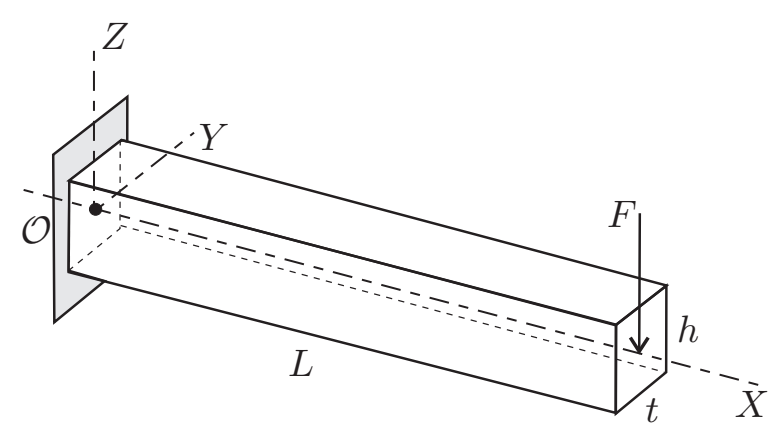

Fig. 2. The cantilever under free-end vertical force.

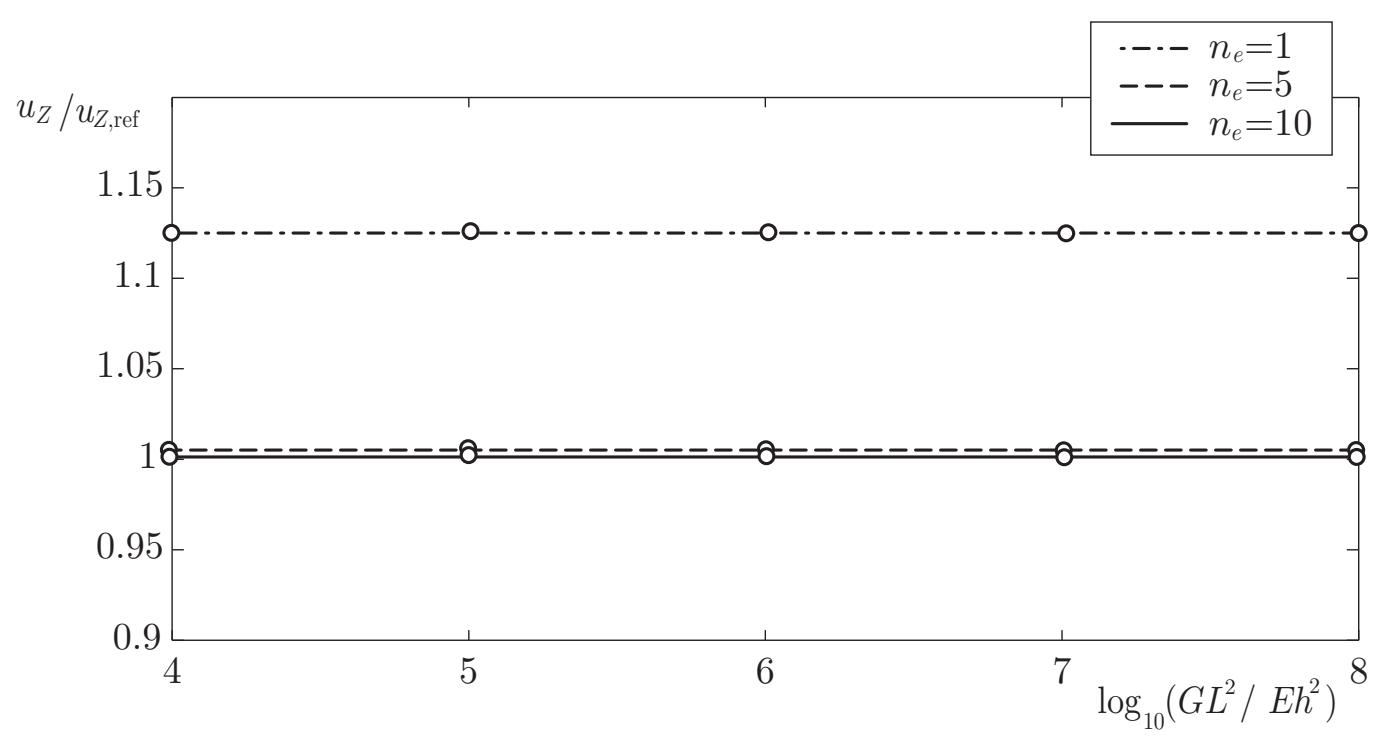

Fig. 3. Normalized vertical tip displacement vs. parameter $G L^{2} / E h^{2}$.

The present results are completely insensitive to the variation of structural parameter for any density of the finite-element mesh. This clearly shows that the present formulation is locking-free.

\subsection{Cantilever beam under free-end moment}

We consider a straight in-plane cantilever beam, subjected to a point moment at its free end (Figure 4). Our numerical results are compared with the analytical solution [26] and the convergence study of the numerical solution is presented. The following geometric and material properties of the cantilever 
are taken into account:

$$
\begin{aligned}
E & =2.1 \cdot 10^{4} \quad G=1.05 \cdot 10^{4} \quad L=100 \\
A_{1} & =20 \quad A_{2}=A_{3}=16 \\
J_{1} & =6.4566 \quad J_{2}=1.6667 \quad J_{3}=666.66 .
\end{aligned}
$$

Two different values of the free-end moment were applied: a unit moment $M_{Y}=1$ resulting in small deformation of the cantilever, and $M_{Y}=100$ resulting in large displacements both in $X$ and $Z$-direction.

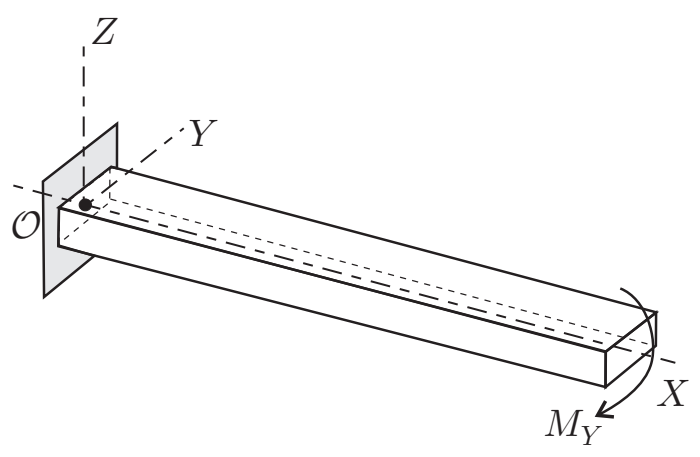

Fig. 4. The cantilever under free-end in-plane moment.

In Table 1 the displacements and the rotation at the free end are displayed and compared to the exact values [26]. Our numerical solution for the freeend rotation agrees with the exact values to all digits for any magnitude of the applied moment regardless of the number and order of elements used. For a small magnitude of the applied moment, even a single element with one internal point gives accurate results. For a large magnitude of the moment, the influence of the non-linearity increases and somewhat affects the accuracy of the discretized solution. It is obvious, however, that in the present case, it suffices to use a single element with two internal points $(N=2)$ to obtain the results accurate to at least five significant digits.

\subsection{Unrolling of circular cantilever}

This example presents a circular in-plane cantilever beam, subjected to a bending moment at the free end (Figure 5). The problem is inverse to a pure bending of a straight cantilever and has an exact solution. By unrolling of a circular beam, we can demonstrate that the finite element properly considers initially curved centroidal axis. The problem was studied by Ibrahimbegović [12]; hence the same geometric and material properties of the beam are taken 
Table 1

Free-end displacements and rotation under an in-plane moment.

\begin{tabular}{|c|c|c|c|c|c|}
\hline & $n_{e}$ & $N$ & $u_{X}$ & $u_{Z}$ & $\vartheta_{Y}$ \\
\hline \multicolumn{6}{|l|}{$M_{Y}=1$} \\
\hline & \multirow[t]{2}{*}{1} & 1 & 0.00010 & 0.14286 & 0.00286 \\
\hline & & 2 & 0.00014 & 0.14286 & 0.00286 \\
\hline & \multirow{2}{*}{$\begin{array}{l}1 \\
2\end{array}$} & 1 & 0.00013 & 0.14286 & 0.00286 \\
\hline & & 2 & 0.00014 & 0.14286 & 0.00286 \\
\hline exact non-lin & 1. $[26]$ & & 0.00014 & 0.14286 & 0.00286 \\
\hline \multicolumn{6}{|c|}{$M_{Y}=100$} \\
\hline & \multirow[t]{2}{*}{1} & 1 & 1.01867 & 14.23718 & 0.28571 \\
\hline & & 2 & 1.35507 & 14.18880 & 0.28571 \\
\hline & \multirow{2}{*}{\multicolumn{2}{|c|}{$\begin{array}{l}1 \\
2\end{array}$}} & 1.27107 & 14.20086 & 0.28571 \\
\hline & & & 1.35501 & 14.18880 & 0.28571 \\
\hline exact non-lin & 1. $[26]$ & & 1.35500 & 14.18880 & 0.28571 \\
\hline
\end{tabular}

here:

$$
\begin{aligned}
& E=1200 \quad G=600 \quad L=10 \\
& A_{1}=1 \quad A_{2}=A_{3}=\frac{5}{6} \\
& J_{1}=0.14058 \quad J_{2}=J_{3}=\frac{1}{12} .
\end{aligned}
$$

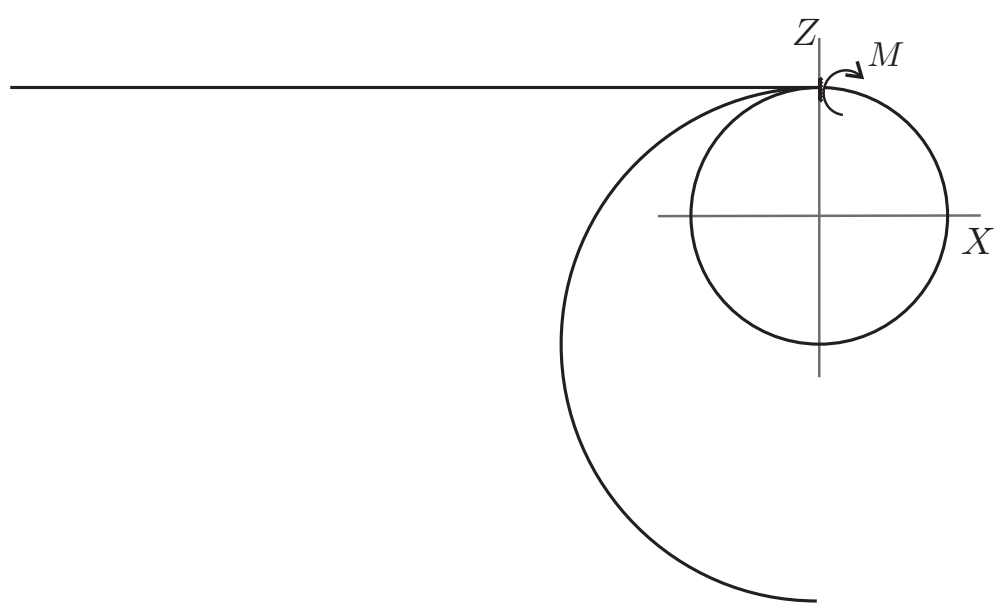

Fig. 5. Unrolling of a circular beam. 
The beam is subjected to the moments $M=10 \pi$ and $M=20 \pi$, which for the chosen characteristics turn the beam into a semicircle and a straight line, respectively. Our results for the free-end displacements and rotation are presented in Table 2 and compared to the exact values. Numerical results are obtained by a mesh of ten curved elements with one or three internal points.

Table 2

Free-end displacements and rotation of the unrolled circular cantilever.

\begin{tabular}{|c|c|c|c|c|}
\hline & $N$ & $u_{X}$ & $u_{Z}$ & $\vartheta_{Y}$ \\
\hline \multicolumn{5}{|c|}{$M_{Y}=10 \pi$} \\
\hline & 1 & 0.04252 & -6.35635 & 3.12505 \\
\hline & 3 & -0.00033 & -6.36645 & 3.14147 \\
\hline exact & & 0.00000 & -6.36620 & 3.14160 \\
\hline \multicolumn{5}{|c|}{$M_{Y}=20 \pi$} \\
\hline & 1 & -9.99847 & 0.13831 & 6.24919 \\
\hline & 3 & -10.00000 & 0.01114 & 6.28196 \\
\hline exact & & -10.00000 & 0.00000 & 6.28320 \\
\hline
\end{tabular}

Our results are in good agreement with the exact ones. Minor differences stem from the error of the polynomial interpolation of rotational quaternions in the undeformed configuration, but they reduce considerably by the use of higherorder elements.

\subsection{Twisted cantilever}

In order to demonstrate that the present finite-element formulation takes the initial twist of cross-sections into account properly, the standard test by MacNeal and Harder [19] is studied. The beam is clamped at one end and subjected to the unit in-plane and the out-of-plane forces at the other. The centroidal axis of the beam is straight at the beginning, but the rectangular cross-sections are twisted about the centroidal axis, see Figure 6 . The initial twist angle along the beam is taken to be a linear function of the arc-length, $x$, with its value set to 0 at the clamped end and to $\frac{1}{2} \pi$ at the free end of the beam. As discussed by Zupan and Saje [35], such a variation of the twist results in curved edges along the length of the beam (Figure 6). The remaining geometric and material characteristics of the beam are:

$$
h=1.1 \quad t=0.32 \quad L=12 \quad E=29 \cdot 10^{6} \quad \nu=0.22 .
$$




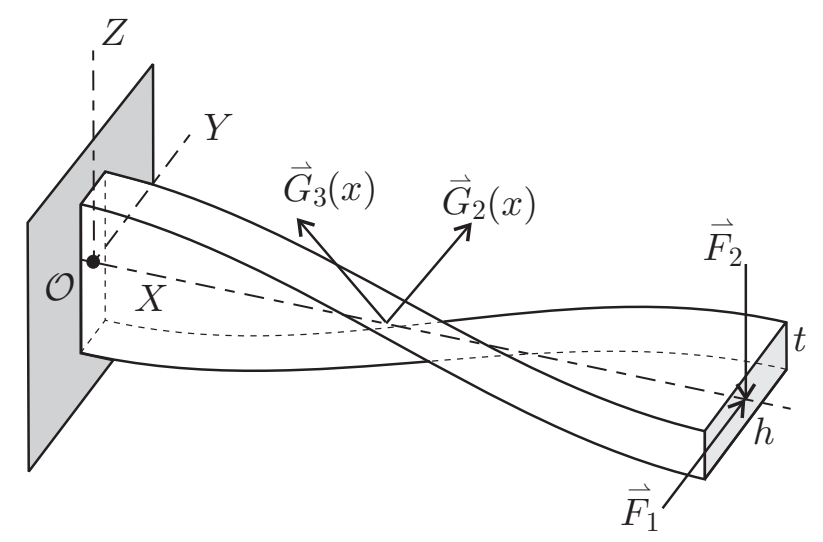

cross section:

Fig. 6. Twisted beam.

Table 3

Free-end displacements of a $\pi / 2$-twisted cantilever.

\begin{tabular}{lrrrrr}
\hline \hline \multicolumn{4}{c}{$F_{1}$} & \multicolumn{2}{c}{$F_{2}$} \\
\hline$n_{e}$ & $N$ & $u_{Y}$ & $u_{Z}$ & $u_{Y}$ & $u_{Z}$ \\
\hline \hline 1 & 3 & 0.005005 & 0.001584 & 0.001431 & 0.001677 \\
& 5 & 0.005427 & 0.001725 & 0.001725 & 0.001754 \\
& 6 & 0.005430 & 0.001720 & 0.001719 & 0.001749 \\
& 7 & 0.005429 & 0.001719 & 0.001719 & 0.001749 \\
\hline 2 & 2 & 0.005428 & 0.001728 & 0.001655 & 0.001711 \\
& 3 & 0.005426 & 0.001716 & 0.001714 & 0.001747 \\
& 4 & 0.005429 & 0.001719 & 0.001719 & 0.001750 \\
\hline 4 & 1 & 0.005480 & 0.001795 & 0.001795 & 0.001755 \\
& 2 & 0.005429 & 0.001719 & 0.001715 & 0.001747 \\
& 3 & 0.005429 & 0.001719 & 0.001719 & 0.001750 \\
\hline \hline \multicolumn{2}{c}{ analytical [36] } & 0.005422 & 0.001719 & 0.001719 & 0.001743 \\
\hline \multicolumn{2}{l}{ theoretical [19] } & 0.005424 & & \multicolumn{3}{c}{0.001754} \\
\hline \hline
\end{tabular}

The results for the free-end displacements in the direction of the applied force are shown in Table 3 and compared to the theoretical values presented by MacNeal and Harder [19]. Their solution is based on the beam equations, which do not fully agree with the present geometrically exact ones. That is why our results are also compared to the analytical solution by Zupan and Saje [36] obtained from the linearized version of the three-dimensional Reissner beam theory.

The initial twist of the cantilever results in displacements being not parallel 
to the applied forces. Numerical results for the free-end displacement indicate that a rather low number of elements is capable of giving highly accurate results. The present results for displacements in the direction perpendicular to the applied force fully agree with the analytical values, while small differences in the parallel direction can be observed. The results accurate in two significant digits are obtained by a single element with five internal points or by four elements with two internal points.

\subsection{Lateral buckling of a right-angle frame}

In this classical problem, introduced by Argyris et al. [2] and later on studied by many others, we analyze a simply supported thin rectangular right-angle frame to check the ability of the present formulation to consider the torsionalbending coupling properly. The frame is subjected to the in-plane moments in opposite directions, as shown in Figure 7 . The same geometrical and material parameters are taken as in [2]:

$$
\begin{array}{lll}
J_{1}=2.16 & A_{1}=18 & E=71240 \\
J_{2}=0.54 & A_{2}=21.6 & G=27191 \\
J_{3}=1350 & A_{3}=21.6 & L=240 .
\end{array}
$$

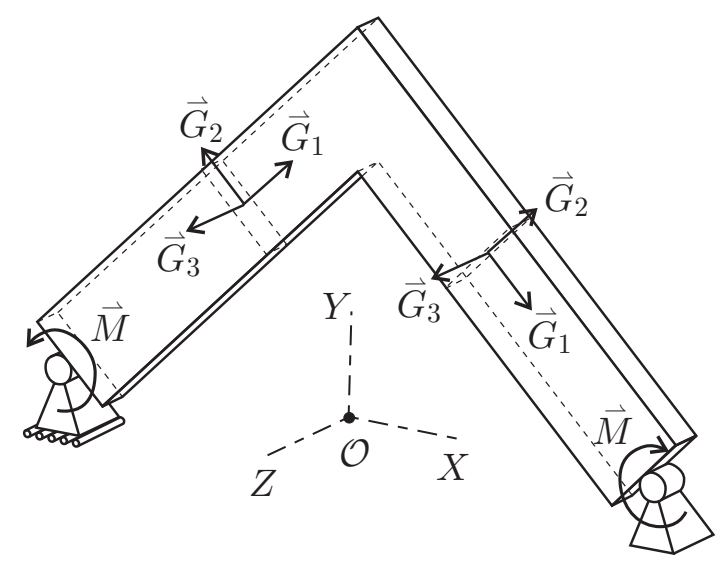

Fig. 7. Lateral buckling of a right-angle frame.

Due to the extreme slenderness of the rectangular cross-section, the frame buckles out of the plane. The numerical value of the critical moment is obtained iteratively from the condition that the tangent stiffness matrix becomes singular. The buckling moment, $M_{c}$, is sought for different-order elements and several finite-element meshes. In Table 4 our results are compared to the an- 
Table 4

Critical moment $M_{c}$ of the simply supported right-angle frame.

\begin{tabular}{|c|c|c|c|c|c|c|}
\hline & $n_{e}=1$ & $n_{e}=2$ & $n_{e}=4$ & $n_{e}=6$ & $\bar{n}_{e}=8$ & $n_{e}=10$ \\
\hline$N=1$ & \pm 622.00 & \pm 622.00 & \pm 622.00 & \pm 622.20 & \pm 622.20 & \pm 622.20 \\
\hline$N=3$ & \pm 622.00 & \pm 622.43 & \pm 622.24 & \pm 622.23 & \pm 622.22 & \\
\hline$N=5$ & \pm 622.19 & \pm 622.22 & \pm 622.22 & \pm 622.22 & & \\
\hline$N=7$ & \pm 622.22 & \pm 622.22 & & & & \\
\hline$[16]$ & \pm 622.2 & & & & & \\
\hline [20] & & & & & & \pm 626.7 \\
\hline [29] & & & & & & \pm 626 \\
\hline analytic & al solution & & & & & 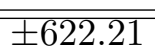 \\
\hline
\end{tabular}

$n_{e}=$ number of elements, $N=$ number of internal points.

alytical solution by Timoshenko and Gere [31] and to the numerical results of others.

The present element gives quite accurate results even for a single low-order element. A rapid convergence of the numerical solution can be observed with the increasing number of internal interpolation points. The four-digit accurate critical moment was obtained with a single element with 5 internal points. The same accuracy can be obtained with 6 elements of the lowest order. It is noted that slight differences between the analytical and numerical solutions are attributed to taking large, but finite values for axial, shear and in-plane bending rigidities rather than the infinite ones, assumed in the analytical solution [31].

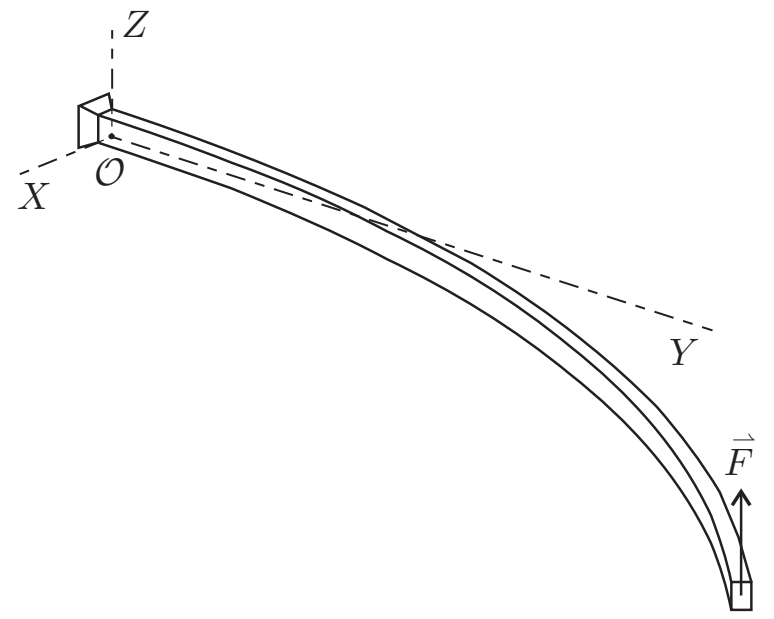

Fig. 8. Cantilever $45^{\circ}$ bend. 


\subsection{Bending of $45^{\circ}$ cantilever}

This classical problem, presented by Bathe and Bolourchi [5], includes all possible natural modes of deformation of a beam: bending, shear, extension and torsion, and has, therefore, become a standard beam finite-element test. The initial axis of the cantilever is an arc of the circle with the radius 100 located in the horizontal plane $(X, Y)$. The cross-section is taken to be a unit square. The beam is subjected to a point load in direction $Z$ at the free end. Two magnitudes of the load are applied: 300 and 600 . The remaining material and geometric data are:

$$
\begin{aligned}
& h=1 \quad t=1 \quad R=100 \\
& E=10^{7} \quad G=E / 2 .
\end{aligned}
$$

No theoretical result is available for this problem. Table 5 displays the comparison of the results for the position vector of the free end of the cantilever. In the majority of solutions given in literature, the arch is modelled with 8 straight elements. Thus, we use 8 straight elements, too, but also compare the results of the mesh made of 8 initially curved elements. The present results were achieved in 6 uniform load steps requiring each 5 iterations for the accuracy tolerance $10^{-9}$. The solutions presented in Table 5 well compare to each other. Only minor differences are observed between the results of the curved and straight elements for the lower value of the applied force. Differences are more significant for the higher value of the load.

Table 5

Free-end position of the cantilever $45^{\circ}$ bend under out-of-plane force.

\begin{tabular}{rrrrrrr}
\hline \hline formulation & $r_{X}$ & $r_{Y}$ & $r_{Z}$ & $r_{X}$ & $r_{Y}$ & $r_{Z}$ \\
\hline present, straight, $N=1$ & 22.16 & 58.55 & 40.52 & 15.91 & 46.98 & 53.94 \\
present, straight, $N=3$ & 22.15 & 58.55 & 40.44 & 15.79 & 46.92 & 53.42 \\
present, curved, $N=1$ & 22.16 & 58.54 & 40.54 & 15.91 & 46.91 & 54.05 \\
present, curved, $N=3$ & 22.14 & 58.54 & 40.47 & 15.61 & 46.89 & 53.60 \\
\hline$[5]$ & 22.5 & 59.2 & 39.5 & 15.9 & 47.2 & 53.4 \\
{$[29]$} & 22.33 & 58.84 & 40.08 & 15.79 & 47.23 & 53.37 \\
{$[8]$} & 22.14 & 58.64 & 40.35 & 15.55 & 47.04 & 53.50 \\
{$[9]$} & 22.31 & 58.85 & 40.08 & 15.75 & 47.25 & 53.37 \\
\hline \hline
\end{tabular}

number of elements $=8, N=$ number of internal points. 


\subsection{Cantilever bent to a helical form}

Our last example considers a straight in-plane cantilever subjected simultaneously to a point moment and an out-of-plane point force at the free end. This example was first presented by Ibrahimbegović [13] and later studied by several authors (e.g. [6], [11], [34]). The analyses of Ibrahimbegović [13] and Battini and Pacoste [6] show the importance of the suitable parametrization of rotations in order to obtain the correct results. This example also demonstrates the ability of parametrization of rotations to consider properly large (more than $2 \pi$ ) and oscillating rotations. The geometric and material properties of the cantilever are:

$$
\begin{aligned}
G A_{2} & =G A_{3}=E A_{1}=10^{4} \quad L=10 \\
E J_{2} & =E J_{3}=G J_{1}=10^{2} .
\end{aligned}
$$

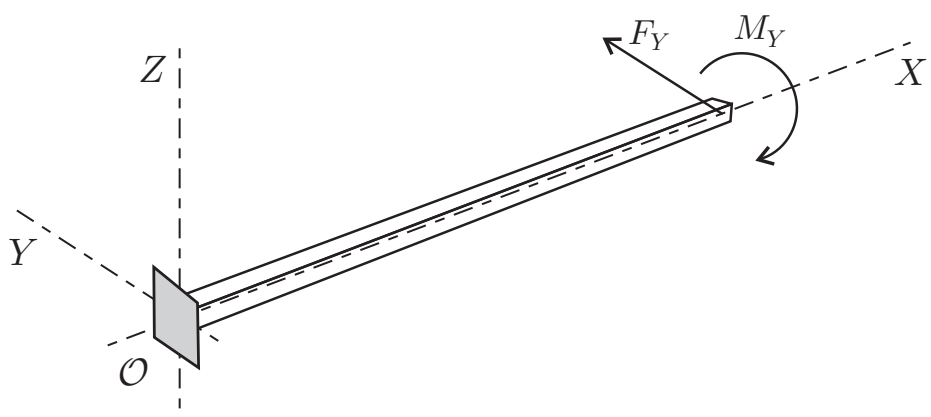

Fig. 9. Cantilever bent to the helical form.

The two loads, $M=200 \pi \lambda$ and $F=50 \lambda$, increase incrementally from $\lambda=0$ to $\lambda=1$ in 1000 steps. The result of a simultaneous application of moment and force is a beam, bent into a helical form. By simultaneously increasing the load, the out-of-plane displacements oscillate around the zero value. For the values of geometrical and material properties as taken, the beam bends into ten helical rings (Figure 10). At the final loading stage, the beam is bent in the direction opposite to the direction of the applied force.

The present results are obtained with the mesh of 25 elements, each with 7 internal points. The displacement $u_{Y}$ of the free end of the cantilever as a function of loading factor $\lambda$ is shown in Figure 11. The results in Figure 11 agree with the results in [13] and are almost identical to the results in [34]. Beam elements presented in [6] and [11] also show very similar behaviour. The above discussed results clearly indicates that large and oscillating rotations present no problem for the present quaternion-based formulation. 


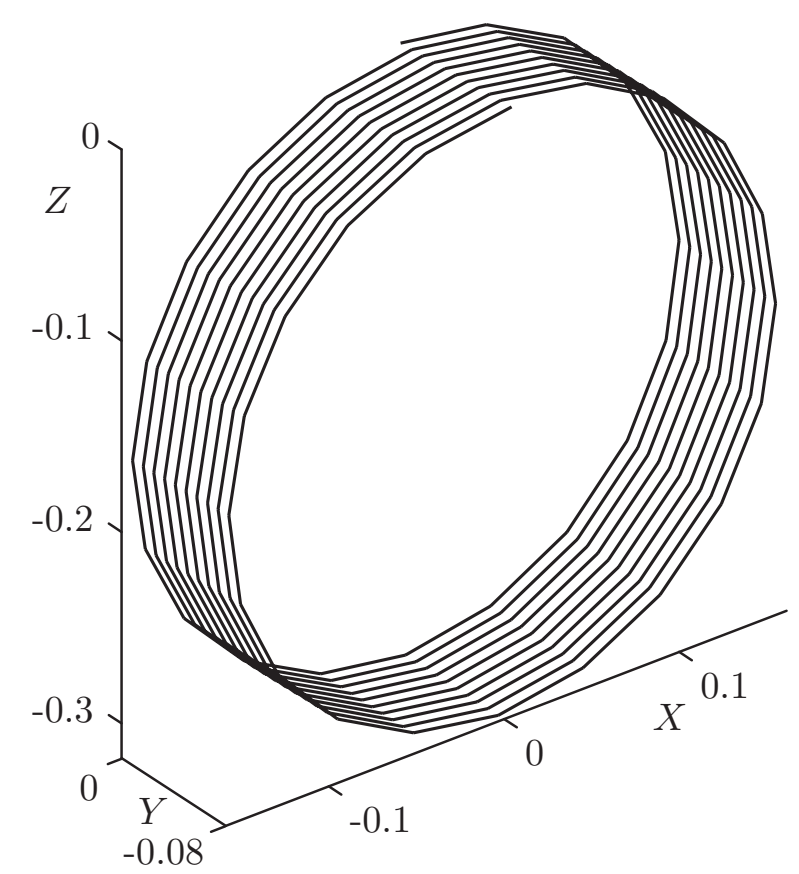

Fig. 10. Cantilever: deformed shape at the final load step.

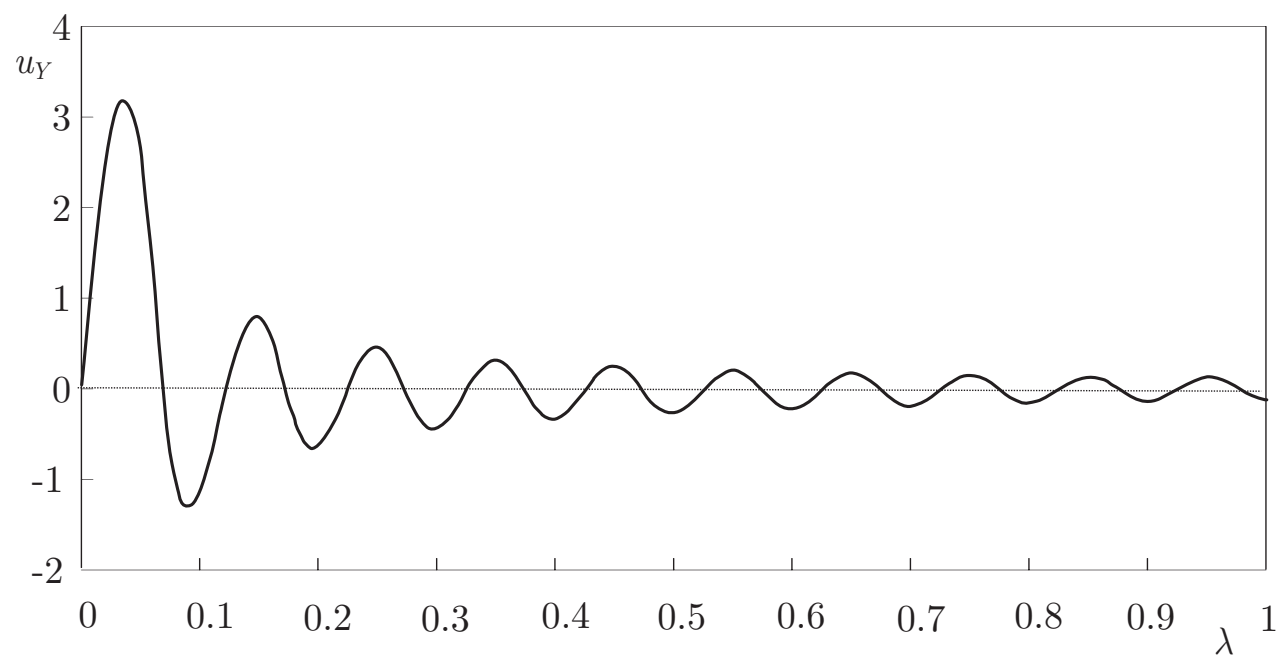

Fig. 11. Load-displacement curve for helical beam.

\section{Conclusions}

The present quaternion-based formulation is specific in several respects. It completely abandons the rotation matrix concept and introduces the rotational quaternions as the rotational measures of the problem. Since the quaternions represent the rotation in the four-dimensional space, it is convenient 
to reformulate the rotational part of the governing equations in the fourdimensional quaternion space, and considering that then the rotational quaternions are mutually independent. This yields the linearization of quaternionbased equations rather simple. Moreover, each of the quaternion variations can be interpolated independently, and, consequently, four iterative increments of quaternion per node become the independent rotational unkowns of the problem. Lagrangian polynomials of the same order for each quaternion component have been employed for the interpolation of iterative increments of quaternions. Such a formulation strictly satisfies the orthonormality condition of the local rotated basis at any point at the beam axis.

The proposed finite-element formulation is also very specific. It is essentially a collocation-type of discretization where the equilibrium conditions for forces and moments are required to be satisfied in strong, integrated form at the two boundary points, while the field equilibrium equations in the differential form are satisfied point-wise at a specified set of interior collocation points. At the midpoint the consistent equilibrium is further required that the constitutive and the equilibrium stress resultants are equal. By such an approach we inherently avoid any shear locking problem for any order of the finite element. In formulating equations at interior points, the constitutive stress resultants are assumed. The present procedures are also fundamental in deriving computational formulae for the derivatives of the rotational quaternion with respect to $x$. It is noted that the above discretization approach can fairly easily be extended for use in dynamic problems.

Numerical experimentations show the validity of the proposed approach for the static geometrically non-linear analysis of initially straight or curved/twisted spatial beams. A large number of various numerical tests performed with new finite elements using different meshes and element orders also confirm their high accuracy, robustness and, due to a favorable number of numerical operations, fast computational performance.

\section{Acknowledgment}

This work was supported by the Ministry of Higher Education, Science and Technology of the Republic of Slovenia through grant 3211-05-000549. The support is gratefully acknowledged. 


\section{References}

[1] J. H. Argyris, "An excursion into large rotations", Comput. Methods Appl. Mech. Eng. 32, 85-155, 1982.

[2] J. H. Argyris, P. C. Dunne, G. Malejannakis, D. W. Scharpf, "On large displacements - small strain analysis of structures with rotational degrees of freedom", Comput. Methods Appl. Mech. Eng. 14, 99-135, 1978.

[3] J. Argyris, V. F. Poterasu, "Large rotations - revisited application of Lie algebra", Comput. Methods Appl. Mech. Eng. 103, 11-42, 1993.

[4] S. N. Atluri, A. Cazzani, "Rotations in computational solid mechanics", Arch. Comput. Methods Eng. 2, 49-138, 1995.

[5] K. J. Bathe, S. Bolourchi, "Large displacement analysis of three-dimensional beam structures", Int. J. Numer. Meth. Eng. 14, 961-986, 1979.

[6] J. Battini, C. Pacoste, "Co-rotational beam elements with warping effects in instability problems", Comp. Methods Appl. Mech. Eng. 191, 1755-1789, 2002.

[7] P. Betsch, P. Steinmann, "Frame-indifferent beam finite elements based upon the geometrically exact beam theory", Int. J. Numer. Meth. Eng. 54, 1775-1788, 2002 .

[8] A. Cardona, M. Géradin, "A beam finite element non-linear theory with finite rotations", Int. J. Numer. Meth. Eng. 26, 2403-2438, 1988.

[9] L. A. Crivelli, C. A. Felippa, "The three-dimensional non-linear Timoshenko beam based on the core-congruential formulation", Int. J. Numer. Meth. Eng. 36, 3647-3673, 1993.

[10] M. Géradin, D. Rixen, "Parametrization of finite rotations in computational dynamics: a review", Revue européenne des éléments finis 4, 497-553, 1995.

[11] S. Ghosh, D. Roy, "A frame-invariant scheme for the geometrically exact beam using rotation vector parametrization", Comput. Mech. 44, 103-118, 2009.

[12] A. Ibrahimbegović, "On finite element implementation of geometrically nonlinear Reissner's beam theory: three-dimensional curved beam elements", Comput. Methods Appl. Mech. Eng. 122, 11-26, 1995.

[13] A. Ibrahimbegović, "On the choice of finite rotation parameters", Comput. Methods Appl. Mech. Eng. 149, 49-71, 1997.

[14] M. Iura, S. N. Atluri, "On a consistent theory and variational formulation of finitely stretched and rotated 3-D space-curved beams", Comput. Mech. 4, 73-88, 1989.

[15] G. Jelenić, M. A. Crisfield, "Geometrically exact 3D beam theory: implementation of a strain-invariant finite element for statics and dynamics", Comput. Methods Appl. Mech. Eng. 171, 141-171, 1999. 
[16] G. Jelenić, M. Saje, "A kinematically exact space finite strain beam model-finite element formulation by generalized virtual work principle", Comput. Methods Appl. Mech. Eng. 120, 131-161, 1995.

[17] The MathWorks, Inc., "MATLAB, Using MATLAB", Natick, http://www. mathworks.com, 1999.

[18] F. A. McRobie, J. Lasenby, "Simo-Vu Quoc rods using Clifford algebra", Int. J. Numer. Methods Eng. 45, 377-398, 1999.

[19] R. H. MacNeal, R. L. Harder, "A proposed standard set of problems to test finite element accuracy", Finite Elem. Anal. Design 1, 3-20, 1985.

[20] B. Nour-Omid, C. C. Rankin, "Finite rotation analysis and consistent linearization using projectors", Comput. Methods Appl. Mech. Eng. 93, 353384, 1991.

[21] M. A. Omar, A. A. Shabana, "A two-dimensional shear deformable beam for large rotation and deformation problems", Journal of Sound and Vibration 243, 565-576, 2001.

[22] W. F. Phillips, C. E. Hailey, G. A. Gebert, "Review of attitude representations used for aircraft kinematics", J. Aircraft 38, 718-737, 2001.

[23] I. R. Poreous, "Clifford Algebras and the Classical Groups", Cambridge University Press, 1995.

[24] E. Reissner, "On finite deformation of space-curved beams", J. Appl. Math. Phys. 32, 734-744, 1981.

[25] I. Romero, "The interpolation of rotations and its application to finite element models of geometrically exact rods", Comput. Mech. 34, 121-133, 2004.

[26] M. Saje, S. Srpčič, "Large deformations of thin curved plane beam of constant initial curvature", Int. J. Mech. Sci. 28, 275-287, 1986.

[27] K. Shoemake, "Animating rotation with quaternion curves", Computer Graphics (ACM) 19, 245-254, 1985.

[28] J. C. Simo, "A finite strain beam formulation. The three-dimensional dynamic problem. Part I", Comput. Methods Appl. Mech. Eng. 49, 55-70, 1985.

[29] J. C. Simo, L. Vu-Quoc, "A three-dimensional finite-strain rod model. Part II: Computational aspects", Comput. Methods Appl. Mech. Eng. 58, 79-116, 1986.

[30] R. A. Spurrier, Comment on "Singularity-free extraction of a quaternion from a direction-cosine matrix", J. Spacecraft 15, 255, 1978.

[31] S. P. Timoshenko, J. M. Gere, "Theory of Elastic Stability", McGraw-Hill, New York, 1961.

[32] J. P. Ward, "Quaternions and Cayley Numbers", Kluwer Academic Publishers, 1997. 
[33] X. Yun, E. R. Bachmann, "Design, implementation, and experimental results of a quaternion-based Kalman filter for human body motion tracking", IEEE Trans. on Robotics 22, 1216-1227, 2006.

[34] D. Zupan, M. Saje, "Finite-element formulation of geometrically exact threedimensional beam theories based on interpolation of strain measures", Comput. Methods Appl. Mech. Eng. 192, 5209-5248, 2003.

[35] D. Zupan, M. Saje, "On "A proposed standard set of problems to test finite element accuracy": The twisted beam", Finite Elem. Anal. Design 40, 14451451, 2004.

[36] D. Zupan, M. Saje, "The linearized three-dimensional beam theory of naturally curved and twisted beams: The strain vectors formulation", Comput. Methods Appl. Mech. Eng. 195, 4557-4578, 2006.

[37] E. Zupan, M. Saje, "The Influence of Parametrization on Integrating Rotations from Angular Velocities", Proceedings of the Sixth International Conference on Engineering Computational Technology, September 2008. Stirling: Civil-Comp Press, 2008. http://dx.doi.org/10.4203/ccp.89.137

\section{Appendix A. Numerical implementation of the rotational quater- nion and its derivatives}

Let $\widehat{\boldsymbol{q}}=\cos \frac{\vartheta}{2}+\boldsymbol{n} \sin \frac{\vartheta}{2}$ be an arbitrary rotational quaternion. It can be expressed in the form of an infinite sum as

$$
\widehat{\boldsymbol{q}}=\widehat{\mathbf{1}}+\frac{1}{1 !} \frac{\widehat{\boldsymbol{\vartheta}}}{2}+\frac{1}{2 !} \frac{\widehat{\boldsymbol{\vartheta}}}{2} \circ \frac{\widehat{\boldsymbol{\vartheta}}}{2}+\frac{1}{3 !} \frac{\widehat{\boldsymbol{\vartheta}}}{2} \circ \frac{\widehat{\boldsymbol{\vartheta}}}{2} \circ \frac{\widehat{\boldsymbol{\vartheta}}}{2}+\ldots=\exp \left(\frac{\widehat{\boldsymbol{\vartheta}}}{2}\right)
$$

where $\widehat{\boldsymbol{\vartheta}}=\left[\begin{array}{ll}0 & \vartheta \\ \boldsymbol{n}\end{array}\right]^{T}$. Expression (99) is called the exponential form of the rotational quaternion. The proof is straightforward: for an arbitrary pure quaternion, $\widehat{\vartheta}$,

$$
\begin{aligned}
\widehat{\boldsymbol{\vartheta}} \circ \widehat{\boldsymbol{\vartheta}} & =-\vartheta^{2} \\
\widehat{\boldsymbol{\vartheta}} \circ(\widehat{\boldsymbol{\vartheta}} \circ \widehat{\boldsymbol{\vartheta}}) & =-\vartheta^{2} \widehat{\boldsymbol{\vartheta}}
\end{aligned}
$$

By inserting (100)-(101) into (99) we get

$$
\begin{aligned}
\widehat{\boldsymbol{q}} & =\widehat{\mathbf{1}}+\frac{1}{1 !} \frac{\widehat{\boldsymbol{\vartheta}}}{2}-\frac{1}{2 !}\left(\frac{\vartheta}{2}\right)^{2}-\frac{1}{3 !} \frac{\vartheta^{2}}{2^{3}} \widehat{\boldsymbol{\vartheta}}+\frac{1}{4 !}\left(\frac{\vartheta}{2}\right)^{4}+\ldots \\
& =1-\frac{1}{2 !}\left(\frac{\vartheta}{2}\right)^{2}+\frac{1}{4 !}\left(\frac{\vartheta}{2}\right)^{4}+\ldots+\frac{\widehat{\boldsymbol{\vartheta}}}{\vartheta}\left(\frac{1}{1 !} \frac{\vartheta}{2}-\frac{1}{3 !}\left(\frac{\vartheta}{2}\right)^{3}+\ldots\right) \\
& =\sin \frac{\vartheta}{2}+\frac{\widehat{\boldsymbol{\vartheta}}}{\vartheta} \cos \frac{\vartheta}{2} .
\end{aligned}
$$


In contrast to its polar form, the expression in equation (99) does not suffer a singularity at $\vartheta=0$. In the numerical implementation, it is therefore preferable to employ (99) for small values of $\vartheta$ at least. The exponential form is also preferable to be used to obtain numerically stable formulae for the derivatives and the variations of the rotational quaternion for small values of $\vartheta$. For example, the first derivative of $\widehat{\boldsymbol{q}}$ with respect to $x$ is achieved by differentiating (99) while taking into account that the quaternion product is non-commutative:

$$
\begin{aligned}
\widehat{\boldsymbol{q}}^{\prime} & =\frac{1}{1 !} \frac{\widehat{\boldsymbol{\vartheta}}^{\prime}}{2}+\frac{1}{2 !}\left(\frac{\widehat{\boldsymbol{\vartheta}}^{\prime}}{2} \circ \frac{\widehat{\boldsymbol{\vartheta}}}{2}+\frac{\widehat{\boldsymbol{\vartheta}}}{2} \circ \frac{\widehat{\boldsymbol{\vartheta}}^{\prime}}{2}\right) \\
& +\frac{1}{3 !}\left(\frac{\widehat{\boldsymbol{\vartheta}}^{\prime}}{2} \circ \frac{\widehat{\boldsymbol{\vartheta}}}{2} \circ \frac{\widehat{\boldsymbol{\vartheta}}}{2}+\frac{\widehat{\boldsymbol{\vartheta}}}{2} \circ \frac{\widehat{\boldsymbol{\vartheta}}^{\prime}}{2} \circ \frac{\widehat{\boldsymbol{\vartheta}}}{2}+\frac{\widehat{\boldsymbol{\vartheta}}}{2} \circ \frac{\widehat{\boldsymbol{\vartheta}}}{2} \circ \frac{\widehat{\boldsymbol{\vartheta}}^{\prime}}{2}\right)+\ldots
\end{aligned}
$$

The second derivative is obtained in an analogous way. 